\title{
High-throughput microscopy exposes a pharmacological window in which dual leucine zipper kinase inhibition preserves neuronal network connectivity
}

\author{
Marlies Verschuuren ${ }^{1 \dagger}$, Peter Verstraelen ${ }^{1 \dagger}$, Gerardo García-Díaz Barriga ${ }^{1}$, Ines Cilissen ${ }^{1,4}$, Emma Coninx ${ }^{2,3}$,
} Mieke Verslegers ${ }^{3}$, Peter H. Larsen ${ }^{4}$, Rony Nuydens ${ }^{4}$ and Winnok H. De Vos ${ }^{1 *}$ (i)

\begin{abstract}
Therapeutic developments for neurodegenerative disorders are redirecting their focus to the mechanisms that contribute to neuronal connectivity and the loss thereof. Using a high-throughput microscopy pipeline that integrates morphological and functional measurements, we found that inhibition of dual leucine zipper kinase (DLK) increased neuronal connectivity in primary cortical cultures. This neuroprotective effect was not only observed in basal conditions but also in cultures depleted from antioxidants and in cultures in which microtubule stability was genetically perturbed. Based on the morphofunctional connectivity signature, we further showed that the effects were limited to a specific dose and time range. Thus, our results illustrate that profiling microscopy images with deep coverage enables sensitive interrogation of neuronal connectivity and allows exposing a pharmacological window for targeted treatments. In doing so, we revealed a broad-spectrum neuroprotective effect of DLK inhibition, which may have relevance to pathological conditions that ar.e associated with compromised neuronal connectivity.
\end{abstract}

Keywords: Neuronal connectivity, Neuronal network, Synapse, Calcium imaging, High-content screening, Neurodegeneration, Antioxidant depletion, hTau.P301L

\section{Introduction}

Wiring the central nervous system demands precise formation and maintenance of neuronal connections. Functional connections between neurons are established by means of micron-sized interfaces, called synapses $[19,44]$. Synaptic activity and the adjoined opening of gated calcium channels generate calcium transients that drive morphological changes such as dendritic growth and arborization, but which can also influence synapse strength [2]. This dynamic remodeling of both neurites and synapses fosters improved communication between neurons allowing synchronous functional activity, thereby

\footnotetext{
* Correspondence: winnok.devos@uantwerpen.be

${ }^{\dagger}$ Marlies Verschuuren and Peter Verstraelen contributed equally to this work.

${ }^{1}$ Laboratory of Cell Biology \& Histology, Department of Veterinary Sciences,

University of Antwerp, Antwerp, Belgium

Full list of author information is available at the end of the article
}

reinforcing the overall connectivity of the neuronal network. All long-lasting adaptations of the brain, including learning, memory, addiction and chronic pain sensation, rely on the continuous remodeling of neuronal network connectivity [2]. And, disruption of this process is a hallmark of numerous neurological diseases, including schizophrenia, major depressive disorder and Alzheimer's disease (AD) [40]. For example, the cognitive impairments witnessed in AD patients correlate with synapse and dendritic loss, as well as with a reduction of the brain activity, indicating an overall decrease in neuronal connectivity $[16,35,60]$. Therefore, therapeutic developments for neurodegenerative disorders are directing their focus to identifying regulators that promote neuronal connectivity or prevent the loss thereof.

The dense, three-dimensional organization and spatial heterogeneity of the brain makes studying neuronal connectivity in vivo a daunting task, which is not amenable to 
upscaling. Therefore, systematic screening efforts most often rely on simplified models such as neuronal cell cultures. Although some immortalized tumor cells (e.g., SH-SY5Y human neuroblastoma cells, NT2 human teratocarcinoma cells, PC12 rat pheochromocytoma cells) can be differentiated to take on a neuron-like phenotype (as evidenced by e.g., neurite outgrowth, expression of synaptic markers, induced or subtle spontaneous calcium activity), none fully recapitulate the full feature set of a physiologically connected neuronal network [1, 11, 18, 23, 27, 29]. Neurons derived from human induced pluripotent stem cell have a much higher translational value in comparison with other cell lines and primary cultures prepared from rodents, but the differentiation process is very labor and time intensive $[15,25$, 36]. That is why, as yet, primary neuronal cultures represent the model of choice for genetic and pharmacological highthroughput screens $[3,6,8,9,28,31,43,53,55]$. However, most of these screens tend to focus on one or two specific readouts such as neuron number $[6,31]$, neurite outgrowth $[3,6,8,31]$ or synapse density $[53,55]$. This reductionist approach can be misleading since it neglects multifactorial effects. For instance, measured changes in synapse density can be a direct reflection of real alterations in the number of synapses [28, 43], but could also be influenced by changes in the neurite density [62]. Furthermore, solely gauging morphological correlates may mask potential changes in functional connectivity. Indeed, it has already been shown that spontaneous activity in primary cultures does not scale linearly with synaptic density [5], and that morphological aberrations of primary neuronal networks do not always result in functional impairments [62]. In order to accurately quantify the overall neuronal connectivity in primary cultures, information gained from several readouts should thus be combined. Hence, instead of using one or a few descriptors, here we comprehensively assess the major morphological and functional correlates of neuronal network connectivity, and we integrate them to accurately map changes between subsequent maturation stages in vitro. In a targeted assay, we identified dual leucine zipper kinase (DLK) inhibition as a positive modulator of neuronal connectivity in unperturbed cultures and as a neuroprotector in cultures grown under suboptimal or compromised conditions.

\section{Materials and methods}

Preparation of primary neuronal cultures and pharmacological treatments

This study was carried out in accordance with the recommendations of the ethical committee for animal experimentation of the University of Antwerp (approved ethical file 2015-54). Hippocampi and cortex were dissected from WT E18 C57Bl6 mouse embryos in Hepes (7 mM)-buffered Hanks Balanced Salt Solution, followed by trypsin digestion $\left(0.05 \% ; 10 \mathrm{~min} ; 37^{\circ} \mathrm{C}\right)$ and mechanical dissociation by trituration through 2 pipette tips with decreasing diameter.
After centrifugation (5 min at $200 \mathrm{~g}$ ), the cell pellet was resuspended in Minimal Essential Medium supplemented with $10 \%$ heat-inactivated normal horse serum and $30 \mathrm{mM}$ glucose. Cells were plated in Poly-D-Lysin-coated 96-well plates (Greiner Cell coat, $\mu$ Clear), at $30,000 \mathrm{cells} / \mathrm{cm}^{2}$, and kept in a humidified $\mathrm{CO}_{2}$ incubator $\left(37^{\circ} \mathrm{C} ; 5 \% \mathrm{CO}_{2}\right)$. After $4 \mathrm{~h}$, the medium was replaced with B27 (2\%) supplemented Neurobasal medium, containing Sodium Pyruvate $(1 \mathrm{mM})$, Glutamax $(2 \mathrm{mM})$, glucose $(30 \mathrm{mM})$ and PenStrep $(0.5 \%)$. For anti-oxidant deprivation, the commercially available B27 supplement minus antioxidants was used. To suppress proliferation of non-neuronal cells, $1 \mu \mathrm{M}$ arabinosylcytosine (AraC) was added in $25 \mu \mathrm{l}$ Neurobasal-B27 medium at the third day after plating. Cell culture supplies were purchased from ThermoFisher. The following compounds were obtained from the in house Janssen pharmacy unless stated otherwise: rapamycin (Santa Cruz sc-3504), memantine, MK801, SAHA, tubastatin, GNE-8505, GNE-3511. These compounds were added in $25 \mu \mathrm{l}$ Neurobasal-B27 medium at final concentrations of $0.01 / 0.1 / 1 / 10 \mu \mathrm{M}$. Compound treatment was initiated at 0 DIV (compound assay and DLK prevention experiments), 14 DIV (DLK rescue experiments) or 21 DIV (DLK aged cultures experiment), and repeated every three or four days. Control cultures received the same volume DMSO, which was always below $0.1 \%$ (Fig. 1).

\section{Immunocytochemistry}

Cultures were fixed (2\% PFA, $20 \mathrm{~min}, \mathrm{RT}$ ) at indicated time points (DIV) and immunocytochemically labeled for a dendrite marker (Chicken Polyclonal against MAP2, Synaptic Systems 188,006, $0.5 \mu \mathrm{g} / \mathrm{ml}$ ), a presynaptic marker (Guinea Pig Polyclonal against Synaptophysin-1, synaptic systems 101,004, $0.5 \mu \mathrm{g} / \mathrm{ml}$ ), a postsynaptic marker (Mouse Monoclonal against PSD95, ThermoFisher MA1-046, $2 \mu \mathrm{g} / \mathrm{ml}$ ) and a nuclear marker (DAPI, $5 \mu \mathrm{g} / \mathrm{ml}$ ) (Fig. 1). In brief, the cultures were permeabilized with $1 \%$ Triton X-100 in blocking buffer $(0.1 \%$ bovine serum albumin and $10 \%$ normal horse serum in PBS) for $10 \mathrm{~min}$, followed by an overnight incubation with the primary antibodies at $4{ }^{\circ} \mathrm{C}$ in blocking buffer. After washing with PBS, secondary antibodies (Donkey-anti-Chicken-Cy5 / Donkey-antiGuineaPig-Cy3 / Goat-anti-Mouse-AlexaFluorPlus488, $1 \mu \mathrm{g} / \mathrm{ml}$ ) were added for $2 \mathrm{~h}$ at room temperature. Finally, DAPI was applied to the cultures for $10 \mathrm{~min}$ at a concentration of $2.5 \mu \mathrm{g} / \mathrm{ml}$, followed by a PBS wash.

\section{AAV-mediated expression of GCaMP6f and MAPT-P301L}

Functional connectivity was assessed by means of live cell calcium imaging of spontaneous neuronal activity (Fig. 1). At 0 DIV, a genetically encoded calcium indicator (GCaMP6f) along with a nuclear-localized red fluorescent protein (nls-dTomato) was introduced via AAV- 


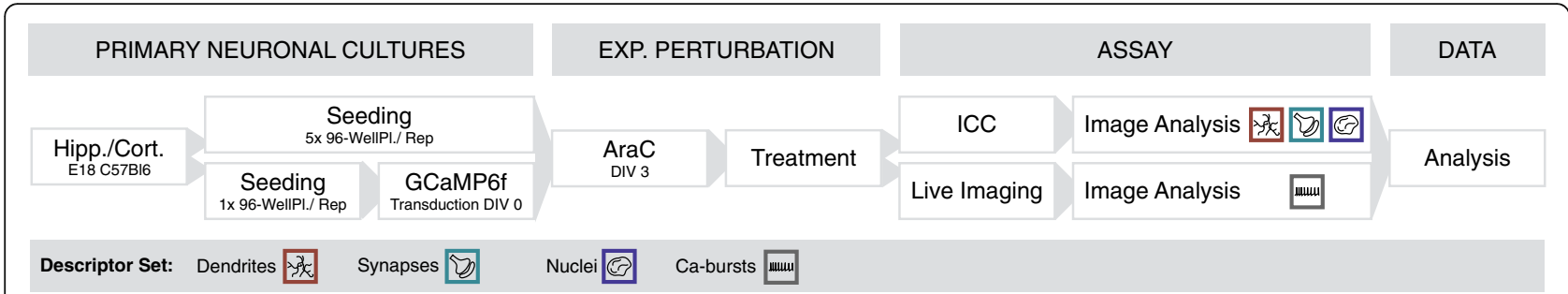

Fig. 1 General workflow. Workflow of the microscopy-based pipeline. Hippocampi and/or cortices from WT E18 C57Bl6 mouse embryos were dissected, after which cell suspensions were created and seeded in 96-well plates. Cultures that would be used in the functional assay were transfected with an AAVDJ-hSyn1-GCaMP6f-nls-dTomato vector on 0 DIV. At 3 DIV, cultures were treated with arabinosylcytosine (AraC) to

suppress the excessive growth of non-neuronal cells. Cultures were treated every three or four days. At fixed time points, cultures were either fixed and subjected to immunostaining, or used for live cell imaging to assess the morphological and functional characteristics, respectively

mediated expression under the synapsin promoter (Addgene plasmid \#51085 deposited by Jonathan Ting was packaged in-house with the AAV-DJ Helper Free Packaging System of Cell Biolabs; $0.01 \mu$ l crude lysate/ well).

Overexpression of hTau.P301L was induced via AAVmediated transduction of an expression vector under the synapsin promotor [7, 59] and were administered at 3 DIV at MOI 300.

\section{Western blot}

Cultures were lysed with ice-cold RIPA buffer (Sigma R0278) supplemented with $5 \mathrm{mM}$ ethylenediaminetetraacetic acid (EDTA) and complemented with phosphatase and protease inhibitors (HALT cocktail, Thermo Scientific \#78445). Protein concentration was measured with the Bicinchoninic Acid Protein Assay kit (Sigma-Aldrich, BCA-1). Lysates were diluted in RIPA buffer to which Cy5 prelabelling was added (Amersham GE Healthcare 29,030,731). After $30 \mathrm{~min}$ on ice a sample mix was added consisting of a 5:2 ratio of NuPage LDS sample buffer (Life Technologies NP0008) and NuPAGE Sample reducing agent (Life Technologies NP00009). Samples were boiled $\left(10 \mathrm{~min}, 70^{\circ} \mathrm{C}\right)$ and $5 \mu \mathrm{g}$ protein per sample was subjected to SDS-PAGE at 100-160 V (Criterion XT BisTris gels, Bio-Rad 345-0124; MOPS SDS Running Buffer, Life Technologies, NP00001). Proteins were transferred to $0.2 \mu \mathrm{m}$ nitrocellulose membranes for $10 \mathrm{~min}$ at 2.5A (Trans-Blot Transfer Pack, Bio-Rad 170-4159; Trans-Blot Turbo Blotting System, Bio-Rad), after which they were gently shaken for one hour in blocking buffer (2.5\% non-fat dry milk (Santa Cruz ChemCruz sc-2325) in Tris Buffered Saline with 0.5\% Tween 20). Next, the membranes were incubated overnight with the following primary antibodies: mouse anti-AT8 (in house JNJ, 1/ 2000), rabbit anti-c-Jun (Cell Signaling Technology \#9165, 1/1000), rabbit anti-p-c-Jun (Ser 63) (Cell Signaling Technology \#9261, 1/500), rabbit anti-p-c-Jun (Ser 73) (Cell Signaling Technology \#3270, 1/500). After washing the blots with Tris Buffered Saline with $0.5 \%$
Tween 20, they were incubated with secondary antibodies for one hour: HRP conjugated donkey anti-rabbit (GE Healthcare Life Sciences NA934, 1/10000) and HRP conjugated sheep anti-mouse (GE Healthcare Life Sciences NA931,1/10000). After a final wash step, the total protein load was detected based on the Cy5 signal using the Amersham AI600 imager. Target proteins were detected by chemiluminescence with Supersignal West Femto Maximum Sensitivity Substrate (Thermo Scientific, 34,076). Images were acquired on the Amersham AI600 imager and analysed using the ImageQuant TL version 8.1 software (GE Healthcare).

\section{Image acquisition}

Confocal images of immunostained cultures were acquired with an Opera Phenix High Content Screening System (PerkinElmer) using 40x water immersion lens (numerical aperture 1.1). At $488 \mathrm{~nm}$ excitation, the optical resolution of the system is $0.271 \mu \mathrm{m}$ (and corresponding pixel size $0.149 \mu \mathrm{m})$, which is considerably larger than the distance of the synaptic cleft $(15-25 \mathrm{~nm})$, yet sufficiently small to allow signals of corresponding pre- and postsynaptic markers to partially overlap [64]. Per well, 15 fields were acquired in 4 channels (405, 488, 561 and $640 \mathrm{~nm}$ excitation) in 3-5 axial positions separated by a $1 \mu \mathrm{m}$ spacing. Different fluorescence channels were separated using standard emission filters and dichroic mirrors.

Calcium imaging was performed on a spinning disk confocal microscope (20x, numerical aperture 0.75 , UltraVIEW VoX, PerkinElmer) at $37{ }^{\circ} \mathrm{C}$ and $5 \% \mathrm{CO}_{2}$. For each field, a 3-min recording (2 frames per second) of the calcium activity was acquired in the $488 \mathrm{~nm}$ channel, followed by a Z-stack of the nuclear NLS-dTomato signal in the $561 \mathrm{~nm}$ channel. Per well, 3 fields were imaged.

\section{Image processing and analysis}

Image analysis was carried out in Acapella ${ }^{\odot}$ (PerkinElmer) based on a template from Evotec, but a similar 
pipeline is available upon request for FIJI [12, 45]. The acquired images were read in per field of view. After maximum projection of the Z-stacks obtained from the MAP2 and DAPI channel, the nuclei were detected using a manually assigned threshold. Dendrites were identified using a rough (user-defined threshold) and fine (user-defined threshold after Frangi filtering [21]) segmentation. Neuronal nuclei were distinguished from non-neuronal based on a user-defined maximal projected area, minimal circularity and minimal occupancy in the dendrite mask. For both the dendrite network and nuclei a range of morphological and textural (object- and image-based) descriptors were extracted (Additional file 1: Table S1). Next, the dendrite mask and the neuronal nuclei mask were dilated and subtracted from each other to obtain a search region (i.e., dilated dendrites without neuronal nuclei) in which the pre- and postsynaptic spots were detected. The sharpest slice (based on the highest standard deviation of the intensity) from the presynaptic channel and corresponding postsynaptic channel were used for spot detection. The spots were first enhanced using a difference of Gaussian filter with a user-defined kernel size, after which a user-defined threshold was applied to segment the spots. To minimize noise contributions, only spots larger than 4 pixels were retained for further analysis. The resolution of the microscope setup does not allow determining the exact location of individual markers within a synapse, but this is not the intention of the assay. Instead, the lower resolution was exploited to define synapses as those objects that demonstrate an overlap of at least 1 pixel between the pre- and postsynaptic spots. We validated that the quantification of synapse density was not biased by chromatic aberration or the overlap criterion (Additional file 2: Figure S1). In addition to this object-based colocalization, the Pearson correlation of the pre- and postsynaptic channel was calculated as an intensity-based colocalization metric. Next to morphological and textural descriptors, the density of pre- and postsynaptic spots and synapses was calculated per measure of dendrite length or dentritic network area (Additional file 1: Table S1).

Calcium recordings were analyzed using a homewritten MATLAB script, adapted from [10]. Briefly, individual neurons were segmented based on the NLSdTomato signals, after which per object traces of the GCaMP6f fluorescence intensity over time were generated. After normalization, signal analysis returned parameters such as percentage of active neurons, frequency and amplitude of (synchronous) calcium bursts and burst correlation, which is the average of the Pearson's correlation matrix between all neuron pairs in the field of view.

\section{Data integration and representation}

Data analysis and representation was done in R Studio [13]. CSV files containing the field data were read and merged with the metadata according to the plate layout. Fields from the morphological assay lacking nuclei or dendrites were removed. False nuclear segmentations (e.g. debris) were identified and removed when the projected nuclear area exceeded the mean projected area by 5 standard deviations. After filtering of the data, well averages were calculated for the morphological data and were depicted as technical replicates. Data extracted from the functional assay was not averaged to not further reduce the number of data points and each recording was considered as a technical replicate. For both assays one biological replicate was considered as a primary culture derived from mouse embryos from one single WT C57Bl6 mother mouse. Z-scores of all descriptors were calculated using the mean and standard deviation within each experiment and replicate. PCA analyses were done on these $\mathrm{z}$-scores using the stats::prcomp function in R. Classification was done on the raw data using the randomForest:: randomForest function in $\mathrm{R}$. Both the control cultures of morphological and functional data were split in a training $(2 / 3)$ and test $(1 / 3)$ set. For each classifier the optimal number of trees and descriptors was determined using 10-fold cross validation, after which the optimal settings were used to classify the test set. The same training and test set was used to train and validate a linear discriminant analysis classifier (LDA, MASS::lda function in R).

The calculation of the connectivity score consisted of four steps; 1 ) first, the correlation of all descriptors with the culture age (DIV) was determined for all control conditions and averaged across six separate experiments; 2 ) next, the inter-correlation of the descriptors was calculated in a merged dataset including control conditions and treated cultures; 3 ) descriptors were then ranked according to their average correlation with culture age; 4 ) this ordered descriptor list, was then filtered top-down, by removing those descriptors with an absolute inter-correlation higher than 0.75 (Additional file 3: Figure S2). Channel descriptors that were sensitive to outliers (e.g., intensity metrics) were removed as well. The final connectivity score was calculated as the average $\mathrm{z}$-score of the subset of descriptors using their respective correlation with DIV as weights. When comparing control cultures with perturbed or treated cultures, z-scores were normalized to control or untreated suboptimal conditions within each experiment, replicate and time point.

All the data representations were built using the ggplot2::ggplot function in R. Mean and standard errors were visualized in bar and line plots. Indicated significance levels were based on pairwise Wilcoxon tests with Bonferroni correction, since normality and homoscedasticity of the data could not be assumed based on the Shapiro-Wilk and Bartlett test. 


\section{Results}

Culture age correlates with both morphological and functional changes in primary neuronal networks

When cultured in vitro, primary neurons form dense dendritic networks and develop functional activity, epitomized by a synchronicity of intracellular calcium fluctuations [5, $63,65]$. To determine the temporal evolution of these changes, we cultured cortical neurons for 48 days in vitro (DIV) and quantified a variety of morphological parameters after fixation and immunostaining at 6-day intervals (Fig. 2a, Additional file 4: Figure S3). We thereby made a distinction between descriptors that inform on one of three major categories, namely the dendrite network, the synapse markers, or the nuclei (Additional file 1: Table S1) . Up to 36 DIV, the neuronal network became denser, as exemplified by an increase in dendrite network density (the area of the field of view covered by dendrites) and the number of nodes (branch points) in the network (Fig. 2b). At later time points, the dendrite network deteriorated. The presynaptic spot density - inferred from synapthophysin immunolabeling - increased rapidly up to 18 DIV, after which it stabilized only to decrease after 30 DIV. The postsynaptic spot density - as gauged from PSD-95 immunolabeling - followed a more gradual evolution and reached a maximum at $42 \mathrm{DIV}$. In line with this, the relative number of overlapping pre- and postsynaptic spots, a proxy for synapse density, stabilized at 18 DIV (Fig. 2b). The number of neuronal nuclei (and thus neurons) gradually decreased with culture age. The ratio of neuronal cells versus non-neuronal (glial) cells remained relatively constant until 36 DIV, after which it significantly decreased, predominantly due to a stronger loss of neurons vs. glial cells (Fig. 2b). Thus, this multiparametric analysis showed that cortical neurons develop progressive morphological connectivity up to 36 DIV with the strongest evolution taking place between 3 and 18 DIV.

We next wondered whether this evolution was generic to cultures derived from different brain regions. Therefore, we compared cortical with hippocampal cultures and quantified changes with a higher time resolution (3, 7, 10, 14 and 18 DIV) (Fig. 2c). For both neuronal culture types, we found similar trends in the previously mentioned descriptors (e.g., dendrite density, synapse density, nuclear count) (Fig. 2d), suggesting that they both become morphologically more connected during this culture period. However, there were also culture type-dependent differences. Hippocampal neurons displayed greater tendency to form tight dendrite bundles than cortical neurons. This clustering occurred especially at later time points (Fig. 2c and Additional file 5: Figure S4a). Hippocampal cultures also showed a stronger postsynaptic marker intensity (Additional file 5: Figure S4a). Arguably one of the most striking differences between both cultures, was a lower proportion of neurons in hippocampal cultures and a comparatively higher number of glial cells. Since glial cells display larger, flattened nuclei with less pronounced chromocenters, this was reflected by a higher average area and lower average (spot-like) texture of nuclei in hippocampal cultures (Additional file 5: Figure S4a,b).

Recognizing that morphological measures do not necessarily report on functional changes in connectivity [62], we complemented the morphological analysis with a previously optimized live cell calcium imaging assay [10]. A very similar evolution was found for both culture types, with virtually no synchronous bursting activity expressed as the correlation between calcium bursts of all active neurons in the field of view - at 3 or 7 DIV, and progressively more synchronous activity at later time points (Fig. 2c,d). This was also reflected - albeit to a lesser extent - in other functional descriptors such as the synchronous bursting frequency (Fig. 2d). Thus, we conclude that primary hippocampal and cortical cultures form neuronal networks that become both morphologically and functionally more connected with culture age, at least up to 18 DIV.

\section{Neuronal culture states can be distinguished by their morphofunctional signature}

Having established that both hippocampal and cortical neurons showed distinct, yet consistent morphofunctional changes with culture age, we asked whether we could retrieve this evolution in an unsupervised manner from the extracted descriptors. Hierarchical clustering on the entire data set revealed that each condition disposed of a distinct descriptor profile, with many descriptors showing a high correlation with culture age (47\% of the descriptors had an absolute correlation with DIV >0.5) (Fig. 3a). To navigate more easily through the high-dimensional descriptor space, we applied principal component analysis (PCA). Despite significant variability between individual biological replicates (Additional file 6: Figure S5a), the pooled data showed a clear and reproducible pattern on the combined morphological descriptor set. When plotting the two first principal components, each cell type displayed a distinct trajectory of instances clustered according to culture age (DIV) (Fig. 3b). We also tested PCA on the separate morphological descriptor classes (nuclei, dendrite, synapse), but no single class could unequivocally separate both cell type and culture time as well, indicating that a combined descriptor set yielded the most powerful discriminatory fingerprint (Additional file 6: Figure S5b). PCA of the functional descriptor set did not lead to well-defined trajectories either, most likely due to the fact that synchronous activity only surfaced from 10 DIV onwards (Fig. 3b).

Given the clear separation in PC space, we next asked whether we could use the information, contained within the descriptors, to predict culture age per cell type. To this end, we trained a random forest classifier (RFC) using 

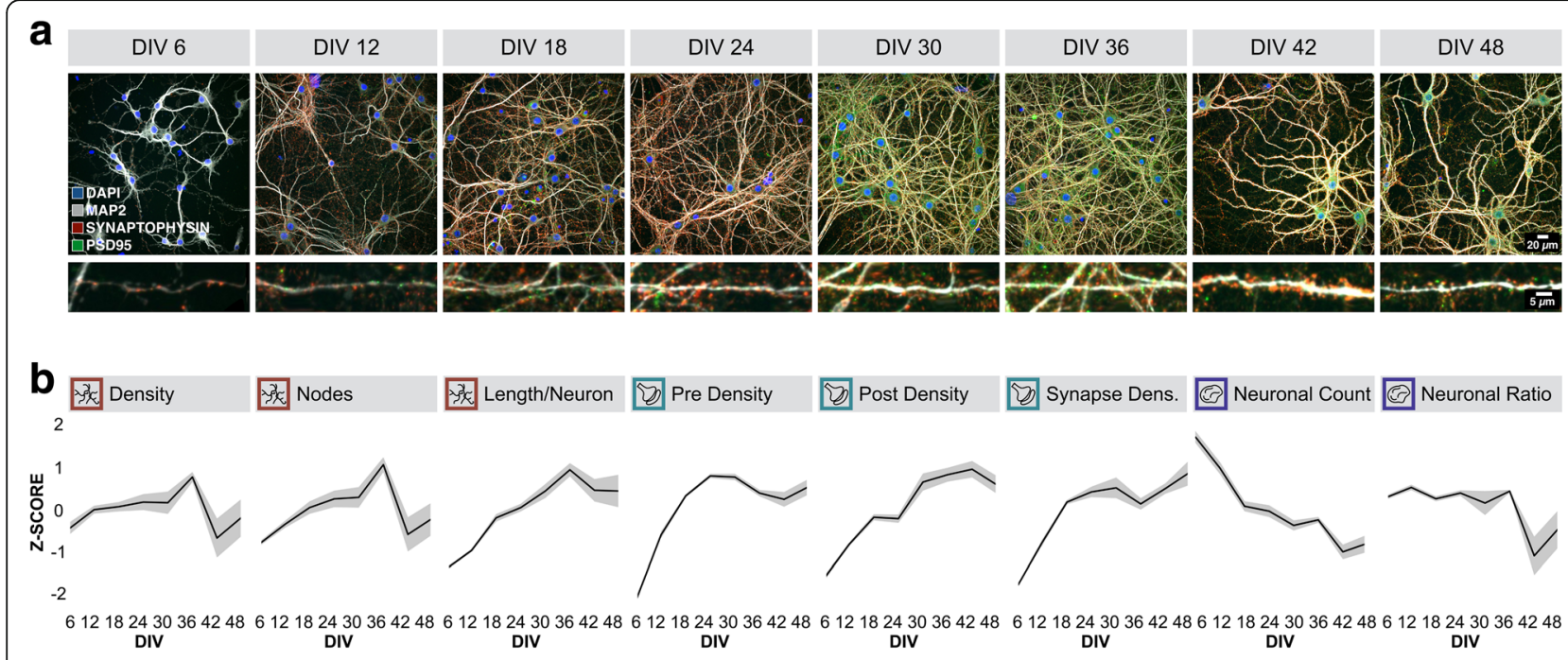

C
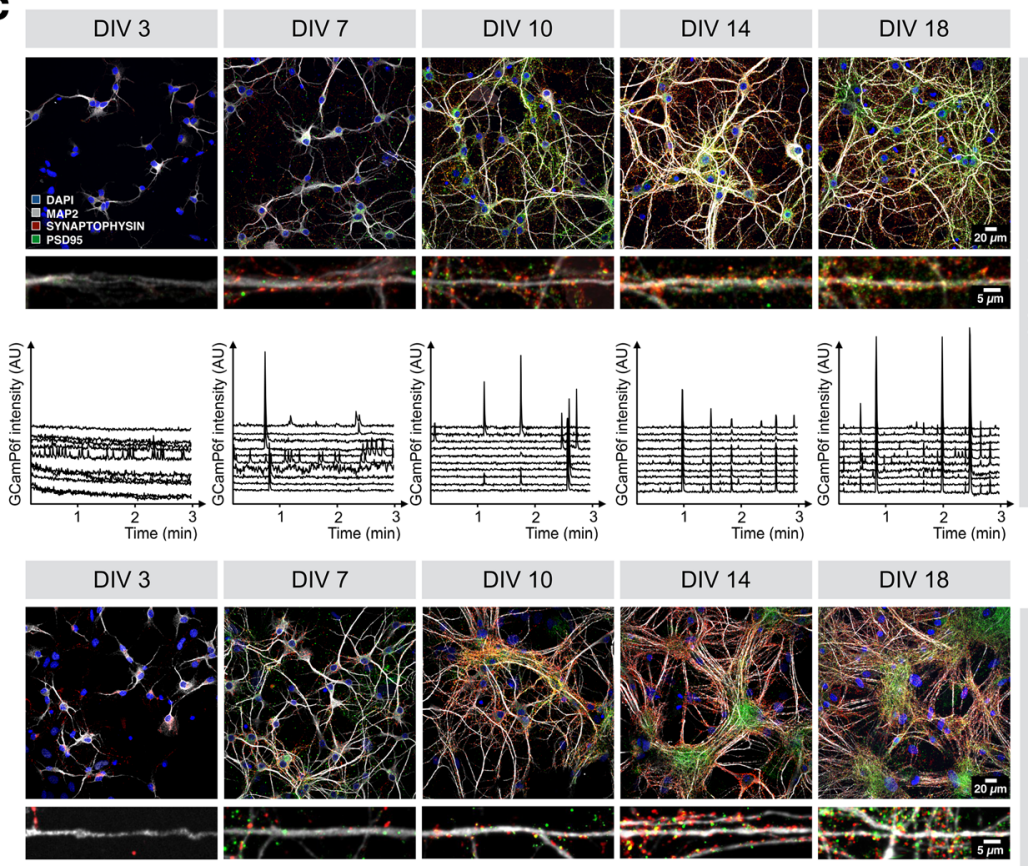

DIV 14

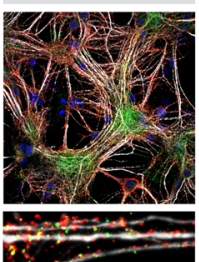

DIV 18
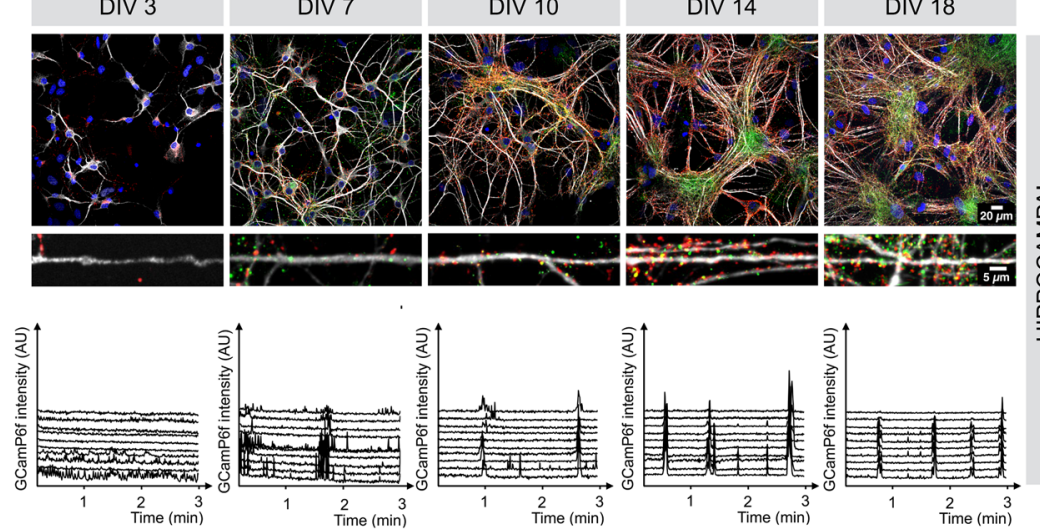

$\frac{8}{8}$

d

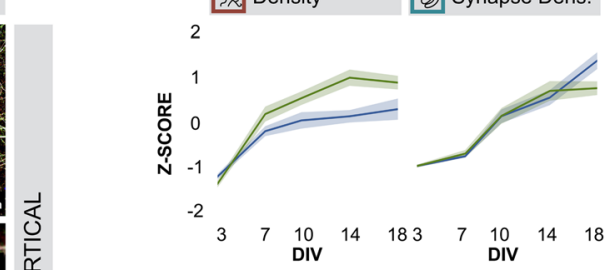

Neuronal Count Neuronal Ratio 2
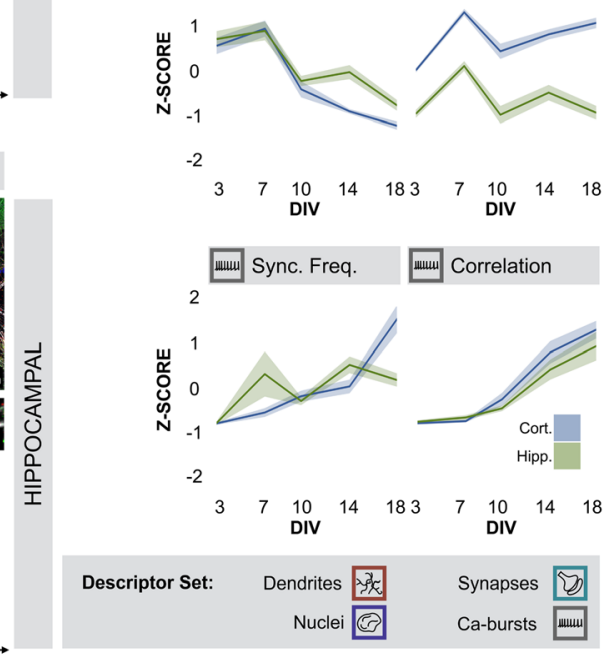

Fig. 2 Culture age correlates with morpho functional changes. a Representative images of cortical cultures after fixation and

immunocytochemistry at 6, 12, 18, 24, 30, 36, 42 and 48 DIV; b Dendrite network density and nodes increased gradually until 36 DIV, after which they decreased. The deterioration of the dendrite network was less pronounced when normalized to the number of neurons, which decreased over time. The presynaptic density and synapse density increased during the first 18 DIV. The density of postsynaptic spots increased gradually. The ratio between neuronal and non-neuronal nuclei remained more or less the same until 36 DIV $\left(n_{\text {bio }}=2 \times n_{\text {tech }}=6\right)$; $\mathbf{c}$ Representative immunocytochemistry images and $\mathrm{Ca}^{2+}$ - traces (of 10 neurons) for a more resolved experiment (3, 7, 10, 14, 18 DIV) of primary hippocampal and cortical cultures; $\mathbf{d}$ Similar trends are observed in selected morphological descriptors for hippocampal and cortical cultures $\left(n_{\text {bio }}=3 \times n_{\text {tech }}=6\right)$. Synchronous activity increased from DIV 10 onwards $\left(n_{\text {bio }}=3 \times n_{\text {tech }}=6\right)$ 


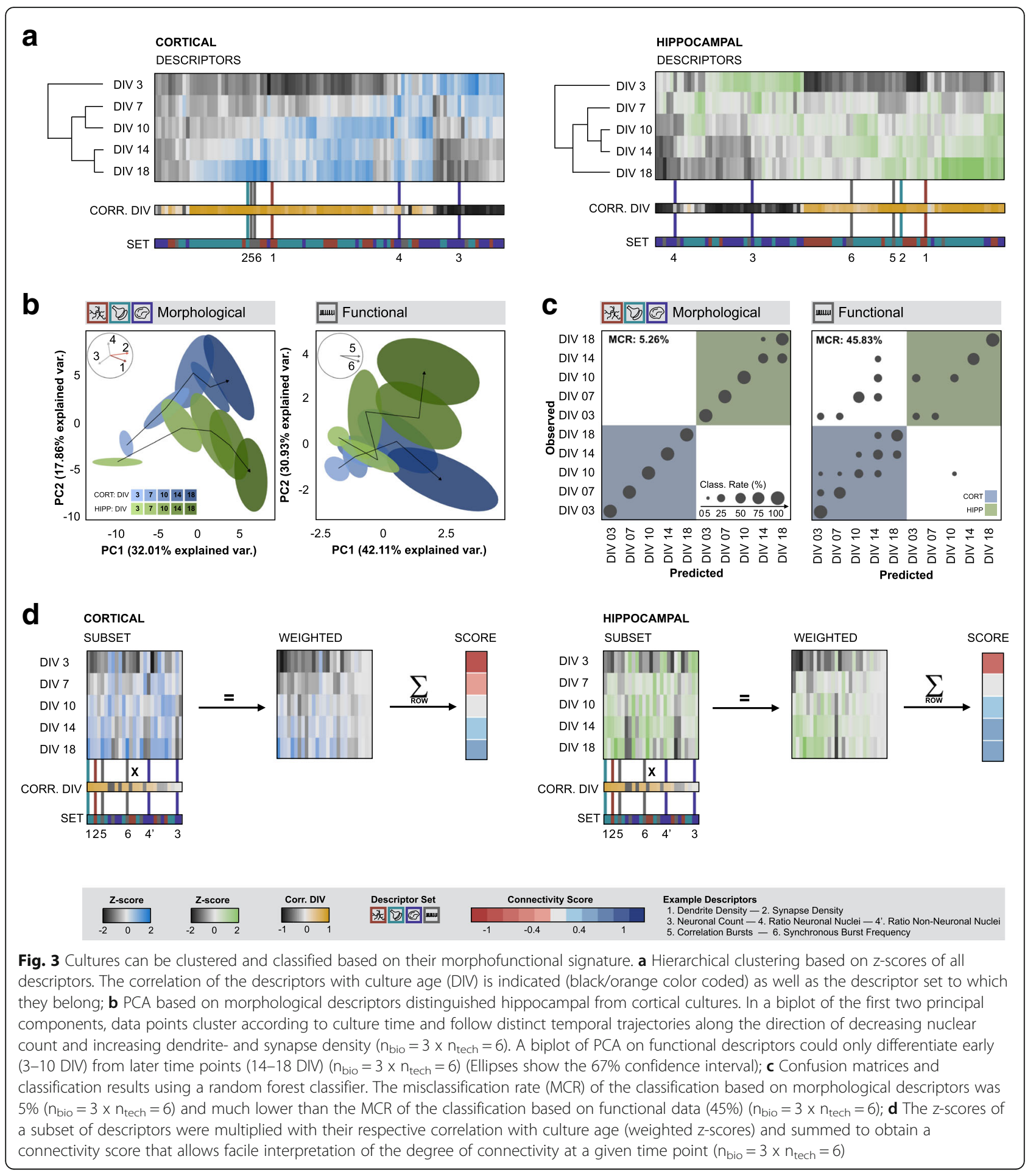

different parameter settings and selected the best performing classifier using 10-fold cross-validation on a training set. Then, we determined the misclassification rate (MCR) on a test set (Fig. 3c). While individual descriptor sets yielded classifiers with MCRs above 14\% (Additional file 6: Figure S5c), a classifier based on all morphological descriptors returned a MCR of 5\%. Comparable results were obtained using a trained linear discriminant analysisbased classifier (Additional file 6: Figure S5d). Not unexpectedly, the performance of a classifier that was solely based on functional descriptors was poor (MCR $>45 \%$ ). Yet, the fact that a morphological descriptor set could be 
used to untangle and even predict culture age, suggests that an integrated signature could be a measure for the degree of neuronal connectivity.

Due to a different experimental setup and number of technical replicates, morphological and functional data could only be combined at the level of the biological replicate. This reduced the number of data points drastically and precluded reliable PCA or RFC. However, we reasoned that the exclusion of functional descriptors from a connectivity analysis could lead to a bias under specific challenged conditions. Indeed, when we treated cortical cultures with the N-methyl-d-aspartate sensitive glutamate receptor (NMDA-R) antagonist MK801, we found an adverse impact on functional connectivity that was significant $(p=9.1 \mathrm{E}-6)$ at $18 \mathrm{DIV}$, without showing significant changes in key morphological descriptors at that time point (dendrite density: $p=1$, synapse density: $p=1$, neuronal count: $p=1$ ) (Additional file 7: Figure S6). Similarly, treatment of hippocampal cultures with arabinosylcytosine (AraC), which selectively blocks astrocyte proliferation, evoked a significant, adverse effect on functional connectivity (burst correlation) at 14 and 18 DIV (14 DIV: $p=2.7 \mathrm{E}-4,18$ DIV: $p=2.5 \mathrm{E}-2$ ), a significant positive effect on dendrite outgrowth at 18 DIV (14 DIV: $\mathrm{p}=1,18$ DIV: $p=4.1 \mathrm{E}-4$ ) and no significant effect on synapse density at 14 and 18 DIV (14 DIV: $p=1,18$ DIV: $p=1$ ) (Additional file 8: Figure S7). The same treatment also caused a significant shift in the non-neuronal nuclei count with culture age (3 DIV: $p=$ 9.9E-9, 7 DIV: $p=1.4 \mathrm{E}-5,10$ DIV: $p=1.0 \mathrm{E}-4,14$ DIV: $p=4.0 \mathrm{E}-8,18$ DIV: $\mathrm{p}=1.4 \mathrm{E}-5)$, suggesting that nuclear descriptors carry relevant information on the culture state as well (Additional file 8: Figure S7). Therefore, we sought an approach to integrate all morphofunctional descriptor classes in such a way that it intuitively reports on connectivity changes, but without disregarding putative off-target effects (Fig. 3d). To avoid redundancy, we excluded descriptors that showed high inter-correlation $(>0.75)$, whilst giving priority to descriptors that correlated best with culture age (Additional file 3: Figure S2). From this subset, a weighted average was calculated using the correlation with culture age as weights. This resulted in a single metric which we refer to as connectivity score (Fig. 3d). The connectivity score is sensitive to changes in any of the four descriptor classes (as demonstrated on the MK801 or AraC data, Additional file 9: Figure S8), allowing it to instantly report on deviations from the basal (unperturbed) connectivity trajectory.

\section{Focused assay identifies DLK as a positive modulator of neuronal network connectivity}

Using the connectivity score as primary readout, we subsequently initiated a focused assay to expose small molecule regulators of neuronal network connectivity. Given the higher culture yield, better reproducibility, and lower tendency to cluster, we chose to continue with cortical cultures. A rational selection of putative molecular targets (mTOR, NMDA-R, histone deacetylases (HDAC), DLK) was made based on their published involvement in neurodegenerative conditions and for each target, at least one compound (rapamycin, memantine, MK801, suberoylanilide hydroxamic acid (SAHA), tubastatin, GNE3511) was selected [24, 38, 41, 46, 51, 56, 72]. Concentrations were based on previously reported $\mathrm{IC}_{50}$ values from neuronal cell-based assays [46, 74-77], within a dose range of $3 \mathrm{log}$ scales. These compounds were tested in unperturbed cultures to expose the dose range that does not exert negative effects in basal conditions. Rapamycin, inhibitor of mTOR, was used as negative control, based on the notion that mTOR activity is crucial in the developmental stages of the neurite network as well as for the synaptic strength at later stages [34]. In total 792.000 images (49.500 fields, 4 channels and $5 \mathrm{z}$-slices) were acquired for this experiment. To reduce variability and aid legibility, scores of challenged conditions were normalized to their culture agematched controls (Fig. 4). As anticipated, rapamycin had an overt negative impact on neuronal connectivity across the tested dose range. Conversely, the DLK inhibitor GNE3511 had an unequivocal positive effect at different culture ages except DIV 18 at doses below $1 \mu \mathrm{M}$. Higher concentrations induced neurotoxicity, which could be expected according to previously reported $\mathrm{IC}_{50}$ values [46]. These findings were also confirmed - albeit to a weaker extent - when using a RFC based on morphological descriptors of the control cultures to predict the degree of connectivity (Additional file 10: Figure S9). Thus, the connectivity score revealed a pharmacological dose and time window in which DLK inhibition promoted connectivity in otherwise unperturbed cultures.

\section{DLK inhibition has both neuro-protective and -restorative potential}

The observation that DLK inhibition exerted a positive effect on neuronal connectivity in primary cultures under basal conditions, drove us to test whether the same treatment was able to prevent or slow down the gradual connectivity loss observed in old cultures (Fig. 5 and Additional file 11: Figure S10). To this end, we incubated cortical cultures with two different DLK inhibitors (GNE3511 and GNE8505) every 3 days from DIV 21 onwards and followed them up to 68 DIV. A positive effect on the connectivity score was found in older cultures (> = DIV48) treated with a low dose $(0.01 \mu \mathrm{M})$ of GNE3511 [46]. Yet, this effect was not consistently recapitulated with GNE8505 [47].

Next, we tested whether DLK inhibition could also prevent cultures from degenerating when grown under sub- 


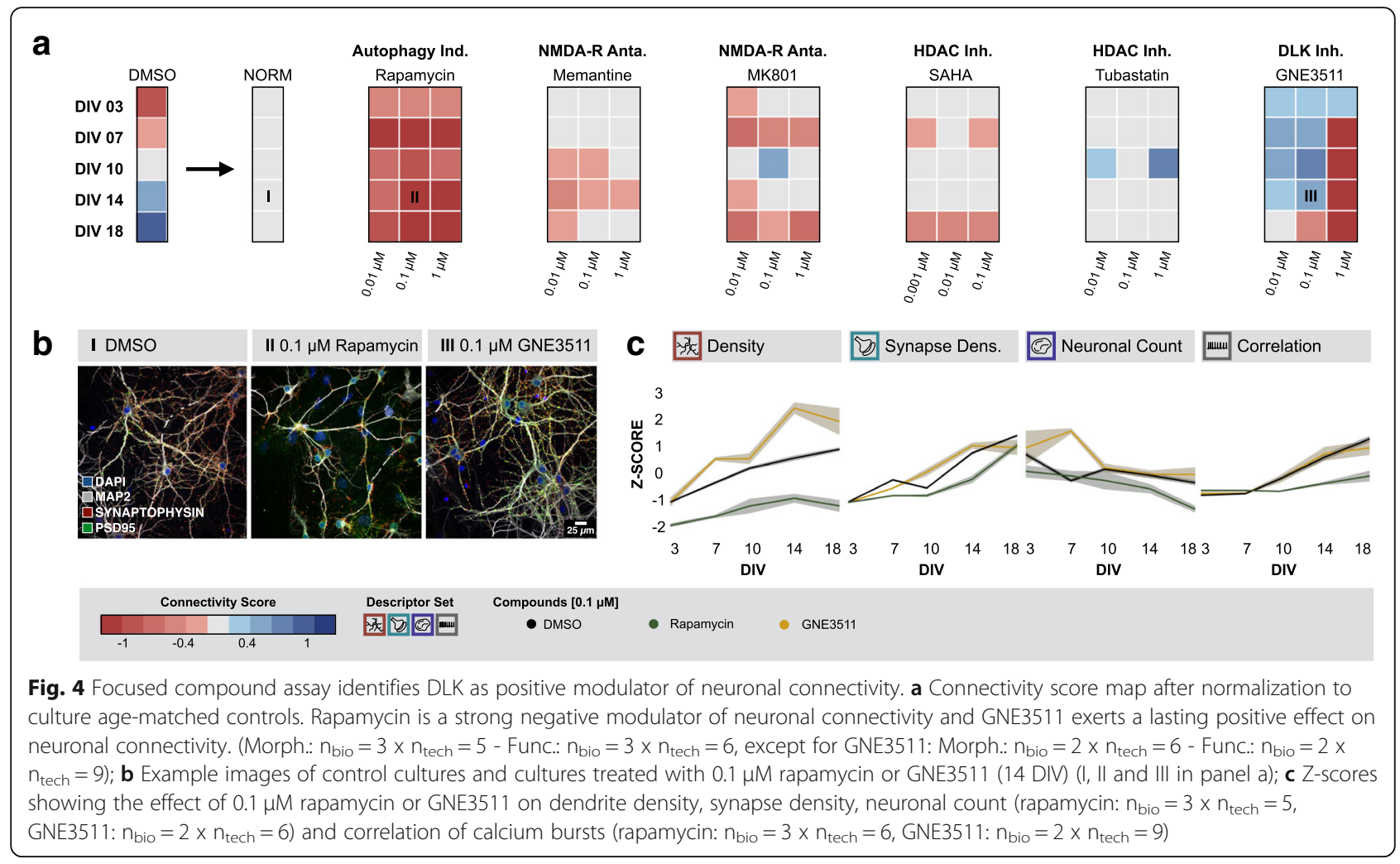

optimal or challenged conditions. Taking advantage of the fact that neurons do not dispose of well-established antioxidant protection mechanisms and are therefore highly susceptible to oxidative stress $[17,61]$, we grew cortical cultures in medium without antioxidants (-AO). This had an adverse impact on neuronal connectivity, as reflected by a decreased connectivity score and increased misclassification by RFC from 7 DIV onwards (Additional file 12: Figure S11ab). To reduce the experimental load, we limited our analyses to one time point where the effect of antioxidant depletion was sufficiently clear, namely at 14 DIV (Fig. 6a). Chronic treatment with $0.1 \mu \mathrm{M}$ GNE3511 could slightly improve the connectivity score in comparison to the DMSO-treated cultures deprived from antioxidants (Fig. 6b), but the increase in the $\mathrm{z}$-score of key morphological descriptors with respect to DMSO-treated -AO cultures was not significant (dendrite density: $p=$ $6.8 \mathrm{E}-1$, synapse density: $p=1$, neuronal count: $\mathrm{p}=1$ )(Fig. 6d). Conversely, chronic treatment with DLK inhibitor GNE8505 $(0.1 \mu \mathrm{M}$ and $1 \mu \mathrm{M})$ drastically improved the connectivity score in comparison with DMSO-treated -AO cultures (Fig. 6b,c). The z-scores of dendrite density, synapse density and neuronal count after treatment with $1 \mu \mathrm{M}$ GNE8505 increased such that they no longer

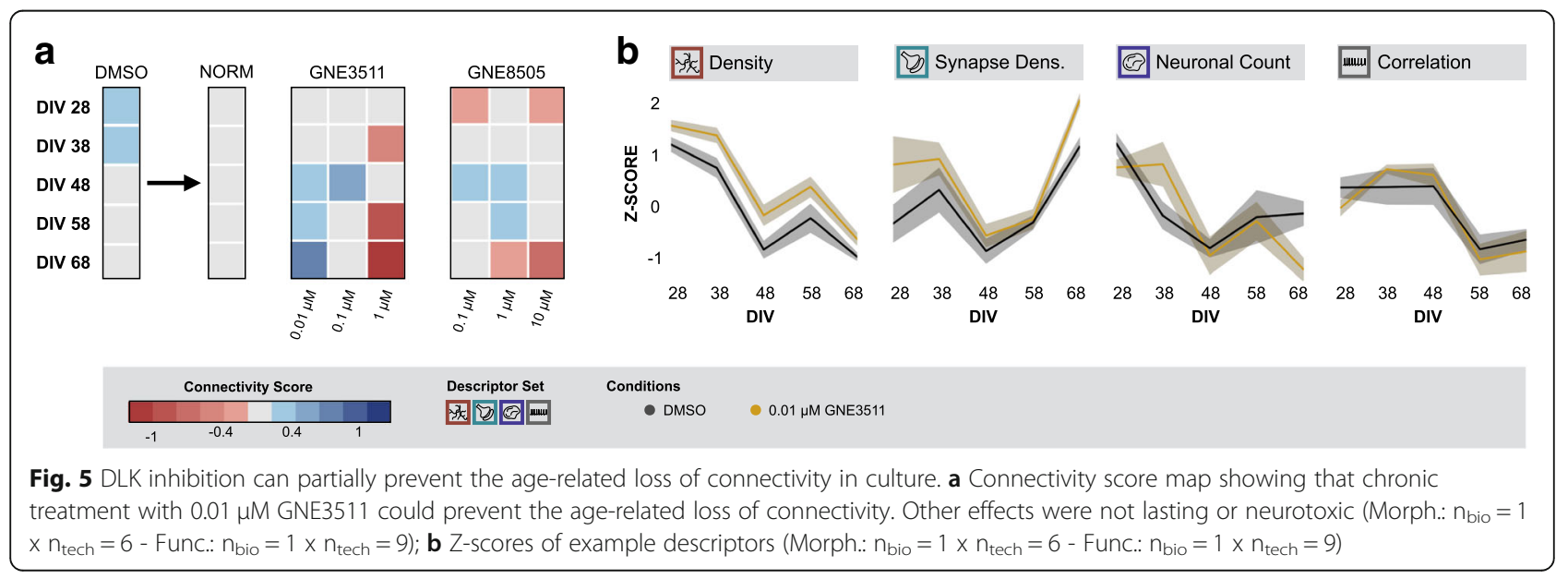




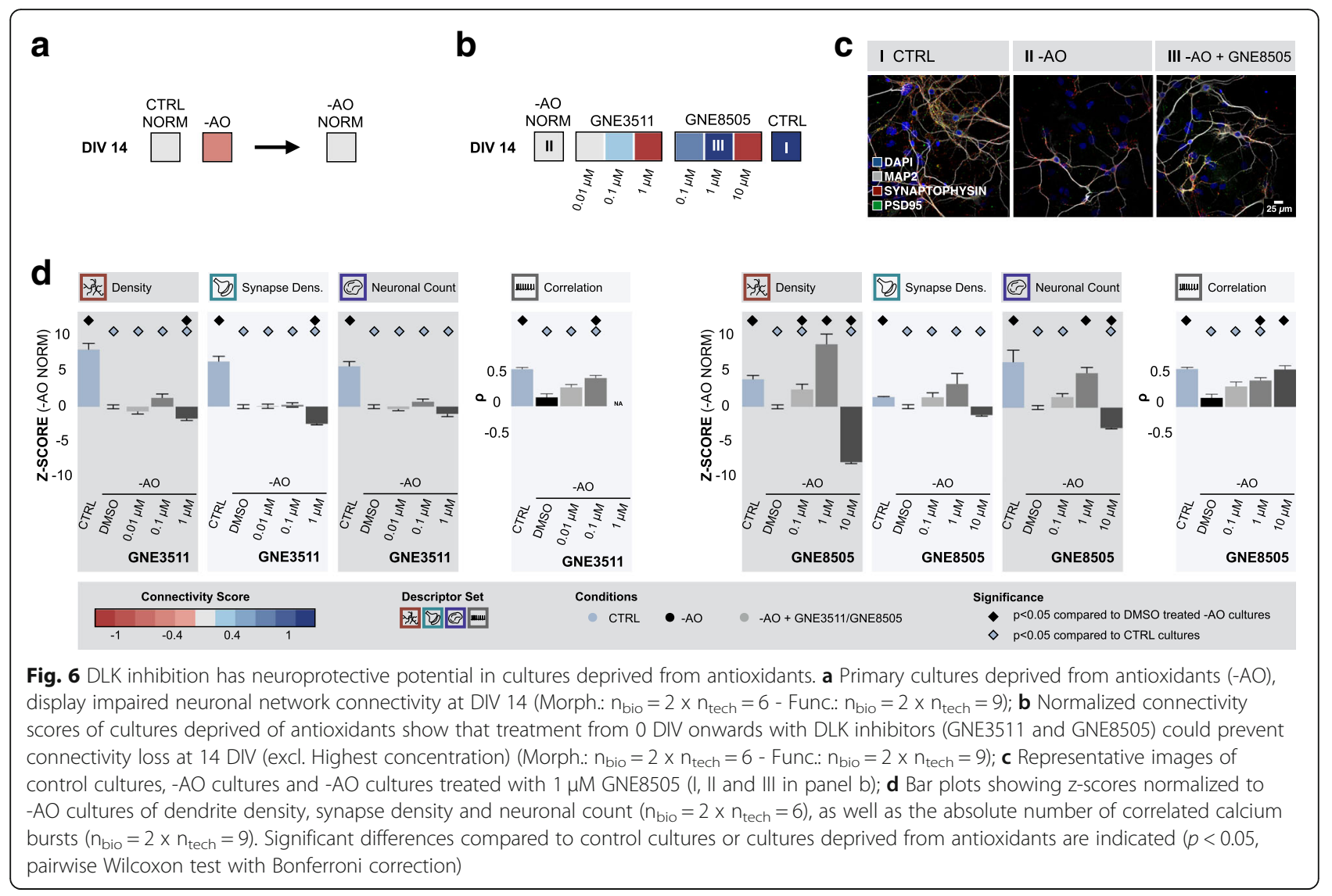

differed significantly from or were higher than those measured in unperturbed control cultures (dendrite density: $p=7.9 \mathrm{E}-1$, synapse density: $p=1.6 \mathrm{E}-2$, neuronal count: $p=4.9 \mathrm{E}-1$ ) (Fig. $6 \mathrm{~d}$ ). These results suggest a neuroprotective effect of GNE8505 in this sub-optimal growth condition. To validate the underlying molecular mechanism, we quantified cJun (a transcription factor in the c-Jun $\mathrm{N}$ terminal kinase (JNK) mediated stress response pathway) levels with western blot. In accordance with the described modus operandi of DLK inhibition [54, 66], GNE8505 lowered both phosphorylated (ser73) and non-phosphorylated cJun levels (Additional file 13: Figure S12a).

To assess the specificity of the neuroprotective action of DLK inhibition, we next introduced a completely different type of challenge to the cultures. We selectively altered microtubule stability through overexpression of hTau.P301L (at 3 DIV). Upon overexpression, a progressive decline in neuronal connectivity was witnessed from 10 DIV onwards (Additional file 12: Figure S11c,d). This correlated with the accumulation of hyperphosphorylated tau, as revealed by AT8 immunoblotting (Additional file 13: Figure S12b) and an upregulation of nonphosphorylated and phosphorylated (ser63) cJun levels (Additional file 13: Figure S12a). When supplementing challenged hTau.P301L cultures with GNE8505, we found a positive impact on the connectivity score in comparison to DMSO treated hTau.P301L cultures at DIV 14 (Fig. 7a,b). After treatment with $1 \mu \mathrm{M}$ or $10 \mu \mathrm{M}$ GNE8505, z-scores of dendrite density, synapse density $(10 \mu \mathrm{M}$ only), and neuronal count showed no significant differences with untreated control cultures (dendrite density: $1 \mu \mathrm{M} p=1$ and $10 \mu \mathrm{M} p=1$, synapse density: $1 \mu \mathrm{M} p=4.4 \mathrm{E}-6$ and $10 \mu \mathrm{M} \mathrm{p}=1$, neuronal count: $1 \mu \mathrm{M}$ $\mathrm{p}=1$ and $10 \mu \mathrm{M} \mathrm{p}=1)($ Fig. $7 \mathrm{c}, \mathrm{d})$, suggesting protection from the genetic insult. In line with - $\mathrm{AO}$ cultures, western blot revealed a significant decrease on both phosphorylated and non-phosphorylated c-Jun levels after GNE8505 treatment (Additional file 13: Figure S12a).

To test whether DLK inhibition could not only prevent but also reverse this process, we repeated the experiments in cultures overexpressing hTau.P301L, but this time we only started treating cultures with DLK inhibitors at a time point where connectivity loss was already manifesting (DIV14). Strikingly, in cortical cultures that overexpressed hTau.P301L, a rescue of connectivity loss could be observed at DIV 18 (Fig. 7e). After treatment of hTau.P301L-overexpressing cells with $10 \mu \mathrm{M}$ GNE8505, dendrite density reached the same level as for untreated control cultures $(p=1.9 \mathrm{E}-1)$ (Fig. 7f,g). Treatment with $1 \mu \mathrm{M}$ GNE3511 even 


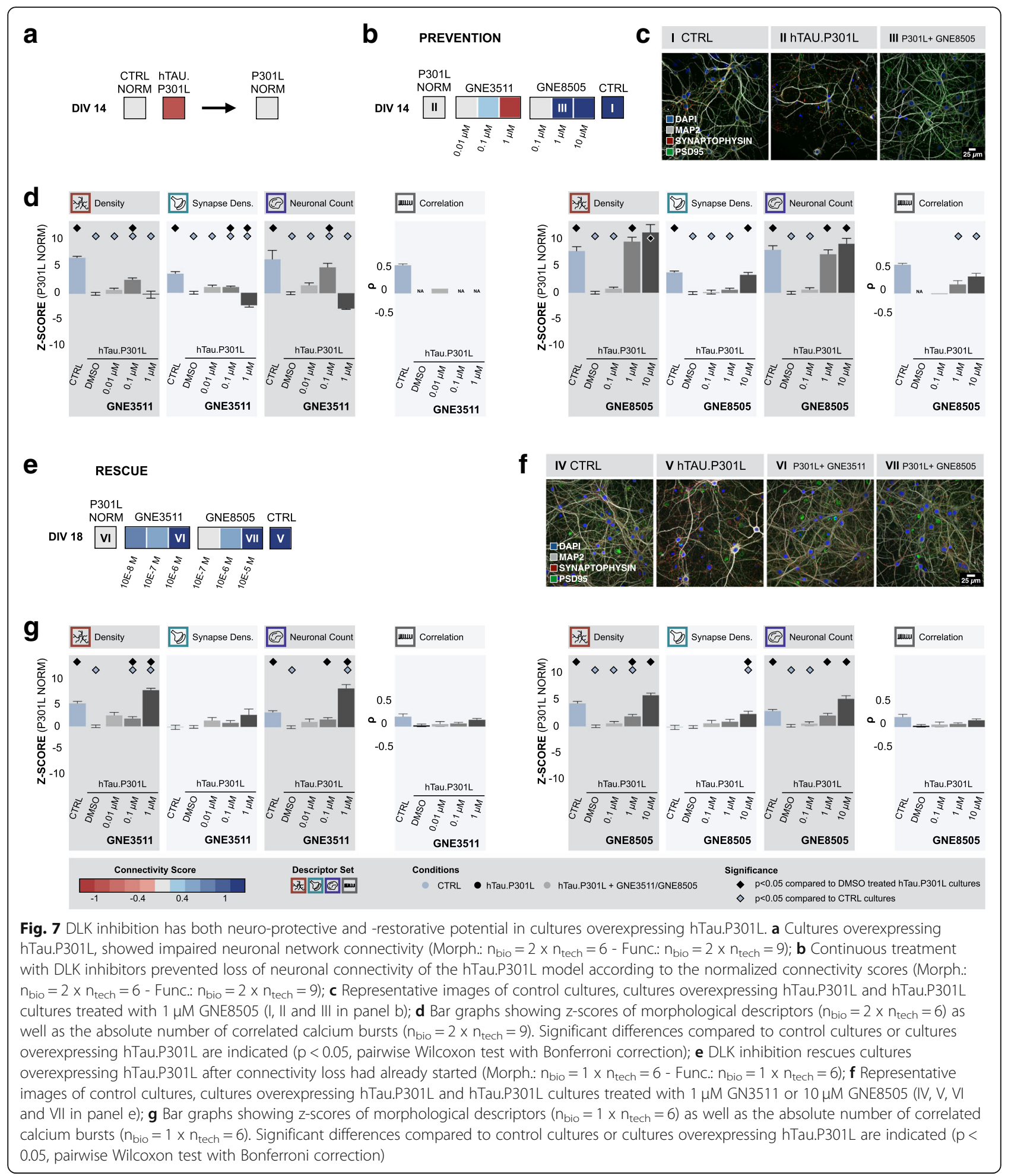

significantly increased the density area above control levels $(p=1.4 \mathrm{E}-3)$ (Fig. $7 \mathrm{f}, \mathrm{g})$. Thus, we conclude that DLK inhibition is able to protect cortical cultures from diverse challenges and even partially rescue cultures from ensuing connectivity loss.

\section{Discussion}

The continuous remodeling of the neuronal network is crucial for learning, memory and behavior, but is disrupted in several psychiatric and neurodegenerative disorders $[40,49]$. Identification of novel therapeutic 
targets requires a method that is able to quantify neuronal network connectivity over time with high accuracy and throughput. To our knowledge, the high-throughput analysis proposed in this paper is the first to comprehensively gauge neuronal connectivity in primary cultures by including descriptor sets reporting on the dendrite network, synapse markers, nuclei and calcium bursting activity. Other high-throughput studies aimed at finding regulators of neuronal connectivity $[3,6,9,28,43]$, have mainly focused at one or few readouts such as neuron number [6] and neurite outgrowth $[3,6]$, synapse density $[53,55]$ or calcium responses [65]. The strength of an integrative approach lies in the fact that it can account for a number of potential sources of bias. For instance, when only considering synapse density, observed changes could be the result of true variations in the synapse number in cultures with comparable dendrite density [28, 43], but they could equally arise from an altered dendrite density with preserved synapse count [62], an increased density of only one or both synaptic partners (pre/post) [43], an increased spot size of one or both synaptic partners, or an altered clustering of neuronal somata and fasciculation of dendrite bundles. Furthermore, the inclusion of calcium data as a proxy for spontaneous electrical network activity allows determining whether alterations in the network morphology also have a functional impact and vice versa. For example, previously published data by our group shows that overexpression of human tau in hippocampal cultures decreased the neurite density but increased the synaptophysin density [62]. At a functional level, the percentage of active neurons and frequency of synchronous bursts decreased while the synchronicity was preserved. This underscores the argument that conclusions may vary depending on the descriptors used and that an integrative approach is desirable.

In line with other studies, we found an increase in dendrite density and synapse density with culture age, and - although not linear - a strong increase in the synchronous bursting behavior of the network at later time points (>10 DIV). The time scales used in literature range from DIV6 to DIV21, which corresponds with the time range we identified in our initial experiment to be the time span in which dendrite density, synapse density and functional activity increased drastically. The decrease in neuronal cell count with culture age confirmed previous findings $[28,33]$, but we now also showed that certain treatments affect cell culture composition, in particular the ratio of nuclear and glial cells (e.g., AraC). To our knowledge, no other connectivity studies have made such a distinction and it shows the value of including nuclear descriptors.

When applying PCA to the unfiltered morphological descriptor set, we unveiled distinct temporal trajectories
[14], suggesting that the culture age could serve as an indicator of connectivity. This was confirmed by the fact that a RFC could predict culture age with an accuracy of 95\%. To include functional parameters in the analysis, we defined a comprehensive connectivity score. Using this score as readout, we confirmed that inhibition of mTOR activity (using rapamycin) impairs the development of the dendritic network [34, 58]. Nevertheless, rapamycin has been used to slow down or block neurodegeneration in mouse models of Alzheimer's, Parkinson's and Huntington's disease, through the induction of autophagy that cleared accumulated autophagosomes and/or aggregated proteins $[4,37,52]$. Therefore, an extension of this work could focus on assessing the effect of rapamycin on neuronal connectivity under conditions of induced toxic protein aggregation. Antagonists of NMDA-R, such as memantine and MK801, were also tested. These antagonists have the potential to block the excessive NMDA-R activity in many neurodegenerative diseases, hereby reducing the increased calcium influx in the neurons $[41,51]$. NMDA-R antagonists can however also block the normal function of these receptors, which was suggested by the overall negative effect on the connectivity score for MK801 (in which the functional descriptors had a major contribution). The score indicated that memantine treatment had only a slight negative impact on neuronal connectivity in comparison with MK801, which may be the result of memantine having a shorter dwell time on the NMDA-R then MK801 [41]. Tubastatin, a HDAC inhibitor that improved cognitive deficits in mouse models of Alzheimer's Disease by improving microtubule stability [72], did not have a negative, nor a neurotrophic effect on the overall neuronal connectivity in control conditions, and may therefore be further explored in compromised conditions. Treatment with another HDAC inhibitor, SAHA, resulted in a decreased neuronal connectivity at later time points. This may be due to the fact that SAHA targets multiple HDAC classes, whereas tubastatin only targets HDAC6 [69].

The sole compound that showed a clear neurotrophic effect in unperturbed primary cultures was an inhibitor of DLK, which is why we chose to pursue the efficiency of this compound in compromised conditions. DLK, also known as MAP3K12, is an upstream regulator of the JNK stress response pathway, which becomes activated in both acute and chronic neurodegenerative conditions [54]. Its activation induces a broad transcriptional injury response (via c-Jun and ATF4) [66]. The fact that it is upstream in this highly conserved pathway makes that DLK inhibition has a broad action range, at least in preclinical models. Pharmacological or genetic DLK inhibition protects against excitotoxicity [50], growth factor deprivation [22, 42, 68], amyloid and tau pathology [32, $38]$, nerve crush and traumatic brain injury [66, 71] 
retinal ganglion cell degeneration $[67,68]$ and SOD1mediated neurodegeneration [38]. At this moment, one inhibitor is in Phase I clinical trial for Amyotrophic Lateral Sclerosis (ALS) by Genentech (Roche) [30]. Our results now show that also in unperturbed primary cortical cultures, DLK inhibition results in enhanced morphofunctional connectivity. Yet, it should be noted that the inhibitor treatment started $4 \mathrm{~h}$ after plating. It is possible that the cells already experienced stress during the dissociation procedure, which could have been attenuated by DLK inhibition.

We found that DLK inhibition could prevent and even rescue neurodegeneration induced by hTau.P301L overexpression. This aligns with literature data where double mutant mice $\left(\mathrm{Tau}^{\mathrm{P} 301 \mathrm{~L}}\right.$; $\left.\mathrm{DLK}^{\mathrm{cKO}}\right)$ show attenuated cell loss in the subiculum compared to single mutant ( $\mathrm{Tau}^{\mathrm{P} 301 \mathrm{~L}}$ ) mice without having altered tau pathology [38]. Our in vitro approach now also shows that hTau-P301L-induced neuron loss is accompanied by impaired dendrite formation, synapse density and functional activity and that this can be prevented and rescued by pharmacological DLK inhibition. DLK inhibition reduced the increased levels of unphosphorylated and phosphorylated (ser63) c-Jun that were detected in cultures overexpressing hTau.P301L.

The omission of anti-oxidants from the culture medium of primary cortical neurons resulted in gradual impairment of morphofunctional connectivity, which could be prevented by DLK inhibition as well. Neurons are particularly sensitive to reactive oxygen species (ROS) and most neurodegenerative disorders are associated with increased oxidative stress [61], making our in vitro model attractive for drug screening. In line with our results, it was described before that the $\mathrm{SOD} 1^{\mathrm{G} 93 \mathrm{~A}}$ mouse model for ALS exhibits neuronal cell death due to an impaired oxidative stress defense and that this is accompanied by aberrant JNK pathway activation [38]. Double mutant mice (SOD1 $\left.{ }^{\mathrm{G} 93 \mathrm{~A}} ; \mathrm{DLK}{ }^{\mathrm{cKO}}\right)$ show enhanced neuronal survival and myelinization as well as reduced neuroinflammation compared to single mutants $\left(\mathrm{SOD} 1^{\mathrm{G} 93 \mathrm{~A}}\right)$, resulting in enhanced grip strength and longer life span of the former model. In AO-depleted cell culture, we did not find evidence for the activation of the JNK pathway, but we did detect a downregulation of the pathway upon DLK inhibition.

Finally, we also verified whether DLK inhibition could sustain morphofunctional connectivity in aging cultures, as it is known that JNK signaling is elevated in the aging brain $[70,73]$. However, the effect was rather limited. Increased variability across aged cultures and reduced sensitivity of the morphological readout due to the very high density of dendrites covering most of the culture plate could have masked a potentially crisper effect of DLK inhibition on ageing. Across experiments, we found differential efficacy of both DLK inhibitors in various growth conditions. These discrepancies are likely the result of different treatment duration and starting times, as well as different target selectivity of both inhibitors $[46,54]$. Literature reports on the selectivity of both compounds show that GNE-3511 had a $\mathrm{K}_{\mathrm{i}}$ (DLK) below $0.5 \mathrm{nM}$ and a pJNK $\mathrm{IC}_{50}$ of $30 \mathrm{nM}$, which was considerably lower than the $\mathrm{IC}_{50}$ for other kinases $(\mathrm{MKK} 4>$ 5.000, MKK7 $>5.000$, JNK1 $=129$, JNK2 $=514$, JNK3 $=$ 364, MLK2 $=767$, MLK3 $=602 \mathrm{nM}$ ), except for MLK1 $\left(\mathrm{IC}_{50}=67.8\right)$ [46]. For GNE-8505, the $\mathrm{K}_{\mathrm{i}}(\mathrm{DLK})$ and pJNK $\mathrm{IC}_{50}$ values were higher than for GNE-3511 $(4 \mathrm{nM}$ and $144 \mathrm{nM}$, respectively), yet the selectivity for DLK over other kinases was markedly better $(\mathrm{MKK} 4>5.000$, MKK7 > 5.000, JNK1 > 10.000, JNK2 > 10.000, JNK3 > 10.000, $\quad$ MLK1 $=3.500, \quad$ MLK2 $=5.150, \quad$ MLK3 $>10.000$ $\mathrm{nM})$. This illustrates that the DLK inhibitors used in this study can target other kinases, especially in the higher dose range, which might explain the differential effects of both compounds.

To increase the sensitivity of the current approach, it could be advantageous to include more synapse markers so as to better map the full landscape of synapse types (e.g., inhibitory vs excitatory). To bypass spectral limitations, one could resort to the use of narrow-emission band labels, such as quantum dots [20], or divert to cyclic immunofluorescence staining protocols $[26,39]$. The latter would however lower the throughput and put a higher demand on downstream analyses (e.g., image registration). A particular caveat of the current approach was the high variability of the functional readout. To strengthen the sensitivity of this assay one could resort to imaging larger cell populations by increasing the field of view. Previous studies have revealed the co-existence of separate networks of high connectivity within a large neuronal network that may not always fire in sync [48, 57] Identification of stratified connectivity patterns may therefore expose more subtle modifications of the functional connectivity. Selective addition of chemical stimuli (e.g., glutamate) could further unveil cell type specificity as well as differences in spontaneous and induced functional activity [10].

\section{Conclusions}

In conclusion, we have shown that morphofunctional profiling of primary cultures using deep coverage microscopy allows accurate quantification of neuronal connectivity in vitro. We established a connectivity score, including morphological and functional correlates, to identify modulators of neuronal connectivity. With our approach we were able to expose a dose and time window for DLK inhibitors that evoked positive effects on neuronal connectivity and could even rescue challenged cultures. Therefore, the current approach holds promise for identifying pathways and 
treatments that preserve or rescue neuronal connectivity in neurodegenerative disorders.

\section{Additional files}

Additional file 1: Table S1. All descriptors for the dendrite network, the synapse markers, the nuclei and functional (calcium) activity. Measurements are reported per field of view. (PDF $10993 \mathrm{~kb}$ )

Additional file 2: Figure S1. Opera Phenix system chromatic aberration and overlap criteria of pre- and postsynaptic markers. (a) Fluorescent beads of 0.1 and $0.5 \mu \mathrm{m}$ diameter were recorded with a 40x water immersion objective $(N A=1.1)$ at an image resolution of $0.149 \mu \mathrm{m} /$ pixel. Dashed white lines show the axis at which the intensities of the $488 \mathrm{~nm}$ (typically used for PSD95) and 561 nm (typically used for Synaptophysin) excitation channel were measured. Quantifications in XY and XZ show that the shift between both channels lies below 1 pixel for both bead types. ( $n=25$ beads); (b) An interpolated shift of 0.6 pixel ( shift calculated for $0.1 \mu \mathrm{m}$ beads in panel (a)) or a one-pixel shift of the PSD95 channel to correct for the chromatic aberration does not alter the synapse density calculation in images of cortical cultures, fixed at 3/7/10/14/ 18 DIV $\left(n_{\text {tech }}=6\right)$; (c) Different criteria for synapse detection (1, 2 or 3 pixels overlap between pre- and postsynaptic spots) alter the absolute synapse numbers slightly, but do not affect the relative change across DIVs $\left(n_{\text {tech }}=6\right)$. (PDF $\left.10993 \mathrm{~kb}\right)$

Additional file 3: Figure S2. Descriptor selection for the connectivity score. A subset of descriptors was selected based on the correlation with the DIV and inter-correlation between descriptors. First, the descriptors were ranked according to their correlation with the culture age. This rank was used to subsequently add descriptors to the final subset, making sure that the inter-correlation within descriptors of the subset did not exceed 0.75 . Intensity descriptors were excluded from the descriptor set, because they were too sensitive to outliers. (PDF $10993 \mathrm{~kb}$ )

Additional file 4: Figure S3. Culture age correlates with morphological changes. Individual channels and composite of representative images of cortical cultures after fixation and immunocytochemistry at $6,12,18,24$. 30, 36, 42 and 48 DIV. (PDF 10993 kb)

Additional file 5: Figure S4. Differences between hippocampal and cortical cultures. (a) Dendrite width and postsynaptic intensity are higher in hippocampal cultures. The ratio of neuronal nuclei is higher in cortical cultures compared to hippocampal cultures that have more nonneuronal nuclei. This is reflected in the average texture and area of the nuclei, since neuronal nuclei are smaller and have a more spot-like phenotype (stronger texture) than non-neuronal nuclei $\left(n_{\text {bio }}=3 \times n_{\text {tech }}=\right.$ 6); (b) Representative images of both neuronal (red) and non-neuronal (yellow) nuclei in cortical and hippocampal cultures. (PDF $10993 \mathrm{~kb}$ )

Additional file 6: Figure S5. Despite inter-replicate variability, cultures can be clustered and accurately classified. (a) Representative images of both hippocampal and cortical cultures at different DIV for 3 different replicates; (b) PCA based on different descriptor sets for individual replicates. PCA based on the integrated descriptor set resulted in the best clustering $\left(n_{\text {bio }}=3 \times n_{\text {tech }}=6\right)$ (Ellipses show the $67 \%$ confidence interval); (c) Confusion matrices of classification results using a random forest. The misclassification rate (MCR) was lowest using the integrated morphological descriptor set $\left(n_{\text {bio }}=3 \times n_{\text {tech }}=6\right)$; (d) Confusion matrices of classification results using a linear discriminant analysis. The MCR, compared to RFC, was lower for morphological data, but higher for functional data $\left(n_{\text {bio }}=3 \times n_{\text {tech }}=6\right)$. (PDF $\left.10993 \mathrm{~kb}\right)$

Additional file 7: Figure S6. Functional descriptors entail unique information about neuronal connectivity. MK801 treatment (yellow) impaired the functional activity significantly at 18 DIV, that was not reflected in the morphological data (Morph.: $n_{\text {bio }}=3 \times n_{\text {tech }}=5$ - Func: $n_{\text {bio }}=3 \times n_{\text {tech }}=6$ ). Significant differences between control and treated cultures are indicated $(p<0.05$, pairwise Wilcoxon test with Bonferroni correction). (PDF $10993 \mathrm{~kb})$

Additional file 8: Figure S7. Nuclear descriptors entail unique information. AraC treatment (yellow) had a major negative impact on nuclear descriptors during the whole time range, while other descriptors showed only transient effects (e.g., dendrite density) or negative effects on later time points (e.g., correlation of the calcium bursts) (Morph: $n_{\text {bio }}=3 \times n_{\text {tech }}=6-$ Func.: $\left.n_{\text {bio }}=3 \times n_{\text {tech }}=6\right)$. Significant differences between control and treated cultures are indicated $(p<0.05$, pairwise Wilcoxon test with Bonferroni correction). (PDF $10993 \mathrm{~kb}$ )

Additional file 9: Figure S8. Connectivity scores are sensitive to changes in dendrite, synapse, nuclear and functional descriptors. (a) Connectivity scores of MK801-treated cultures showed greater differences with DMSO-treated cultures at later time points when based on the integrated dataset when compared to the scores only based on morphological data. (Morph:: $n_{\text {bio }}=3 \times n_{\text {tech }}=5$ - Func.: $n_{\text {bio }}=3 \times n_{\text {tech }}=6$ ); (b) Connectivity scores of AraC treated cultures revealed greater connectivity impairments in comparison with DMSO treated cultures when including nuclear descriptors (Morph.: $n_{\text {bio }}=3 \times n_{\text {tech }}=6$ - Func.: $n_{\text {bio }}=3 \times n_{\text {tech }}=$ 6). (PDF $10993 \mathrm{~kb})$

Additional file 10: Figure S9. Classification of morphological data confirms findings based on connectivity score. A RFC that was trained on pooled DMSO treated cultures revealed a negative impact of rapamycin on neuronal connectivity as can be seen from the cultures that were misclassified and were assigned a culture age that was lower than the actual culture age (red). Treatment with $0.01 \mu \mathrm{M}$ and $0.1 \mu \mathrm{M}$ of GNE3511 could however improve the neuronal connectivity (green) $\left(n_{\text {bio }}=3 \times\right.$ $n_{\text {tech }}=5$ except for GNE3511: $\left.n_{\text {bio }}=2 \times n_{\text {tech }}=6\right)$. (PDF $\left.10993 \mathrm{~kb}\right)$

Additional file 11: Figure S10. Extended culture age reduces neuronal connectivity. Connectivity scores based on z-scores from cortical cultures grown for an extended period of time. Neuronal connectivity increased during the first two weeks, after which it stagnated up to five and a half weeks. From DIV 38 onwards age-related loss of neuronal connectivity was detected (Morph:: $n_{\text {bio }}=1 \times n_{\text {tech }}=6-$ Func:. $n_{\text {bio }}=1 \times n_{\text {tech }}=9$ ). (PDF $10993 \mathrm{~kb}$ )

Additional file 12: Figure S11. Impaired neuronal connectivity in suboptimal conditions. (a) Connectivity scores indicated that antioxidant deprivation $(-\mathrm{AO})$ in primary cultures had a negative impact on neuronal network connectivity form 7 DIV onwards (Morph.: $n_{\text {bio }}=2 \times n_{\text {tech }}=6$ Func.: $n_{\text {bio }}=2 \times n_{\text {tech }}=9$ ); (b) A RFC that was trained on morphological data of pooled DMSO treated cultures confirmed the negative impact of antioxidant deprivation (red) $\left(n_{\text {bio }}=2 \times n_{\text {tech }}=6\right)$; (c) Cultures overexpressing hTau.P301L, showed a decreasing neuronal connectivity from 10 DIV (Morph.: $n_{\text {bio }}=2 \times n_{\text {tech }}=12$ - Func.: $n_{\text {bio }}=2 \times n_{\text {tech }}=9$ ); (d) Classification results based on morphological data confirmed the negative effect of hTau.P301L overexpression on neuronal connectivity (red) $\left(n_{\text {bio }}=2 \times n_{\text {tech }}=12\right)$. (PDF $\left.10993 \mathrm{~kb}\right)$

Additional file 13: Figure S12. Western blot analyses of (phosphorylated) Jun and AT8. (a) Western blot showed an increase in total c-Jun and phosphorylated c-Jun (Ser 63) in cultures overexpressing hTAU.P301L. Treatment with GNE8505 reduced c-Jun and phosphorylated c-Jun in control, antioxidant deprived (-AO) and hTau.P301L cultures (excl. Phosphorylated c-Jun Ser 63 in control and -AO cultures) ( $n_{\text {bio }}=1 \times$ $\left.n_{\text {tech }}=1\right)$; (b) Western blot showed an increase in hyperphosphorylated (AT8) tau in cultures overexpressing hTau.P301L $\left(n_{\text {bio }}=1 \times n_{\text {tech }}=1\right)$. (PDF $10993 \mathrm{~kb})$

\section{Abbreviations}

AraC: Arabinosylcytosine; DIV: Days in vitro; DLK: Dual leucine zipper kinase; EDTA: Ethylenediaminetetraacetic acid; HDAC: Histone deacetylase; JNK: cJun N-terminal kinase; LDA: Linear discriminant analysis; MCR: Misclassification rate; NMDA-R: N-methyl-d-aspartate sensitive glutamate receptor; PCA: Principal component analysis; RFC: Random forest classifier; SAHA: Suberoylanilide hydroxamic acid

\section{Acknowledgements}

The authors would like to thank Sofie Thys for technical assistance during preparation of primary neuronal cultures and Ilse Lenaerts for the western blot analysis.

\section{Funding}

This study was supported by an R\&D (IWT150003) grant of Flanders Innovation \& Entrepreneurship (VLAIO) and Janssen Pharmaceutica. MV holds a PhD Fellowship (FWO 11ZF116N) of the Flemish Institute for Scientific 
Research. WDV is supported by FWO and Stichting Alzheimer Onderzoek (SAO).

\section{Availability of data and materials}

The datasets generated and/or analyzed during the current study are available from the corresponding author on reasonable request and a subset of the data (Replicate 1 from Fig. 2c,d/ Additional file 5: Figure S4/Additional file 6: Figure S5 and replicate 2 from Fig. 7b, c) is available via the BioStudies database (http://www.ebi.ac.uk/biostudies) under accession number S-BIAD7. The Acapella script that was written to quantify the images acquired with the Opera Phenix system is available on GitHub: (https://github.com/ verschuurenM/NeuronalConnectivity).

\section{Authors' contributions}

PV, M Verschuuren, RN, PL, AE, M Verslegers and WDV conceived and designed the experiments. PV, IC, GG and EC performed the wet lab work. M Verschuuren and WDV devised the image analysis script for neuronal morphology. PV analyzed the imaging data (morphological and functional) with available scripts. M Verschuuren, PV, GG and WDV performed data analysis. PV, M Verschuuren and WDV drafted the manuscript. All authors critically revised the manuscript and are accountable for all aspects of the work. (M Verschuuren, M Verslegers). All authors read and approved the final manuscript.

\section{Ethics approval and consent to participate}

The preparation of primary neuronal cultures was carried out in accordance with the recommendations of the ethical committee for animal experimentation of the University of Antwerp (approved ethical file 2015-54).

\section{Consent for publication}

Not applicable.

\section{Competing interests}

Authors (IC, PL and RN) are employees of Janssen Pharmaceutica NV. The authors declare that they have no potential competing interests.

\section{Publisher's Note}

Springer Nature remains neutral with regard to jurisdictional claims in published maps and institutional affiliations.

\section{Author details}

'Laboratory of Cell Biology \& Histology, Department of Veterinary Sciences, University of Antwerp, Antwerp, Belgium. ${ }^{2}$ Department of Biology, Animal Physiology and Neurobiology, KU Leuven, Antwerp, Belgium. ${ }^{3}$ Radiobiology Unit, Institute of Environment, Health and Safety, Belgian Nuclear Research Centre, Mol, Belgium. ${ }^{4}$ Janssen Research \& Development, a Division of Janssen Pharmaceutica NV, Beerse, Belgium.

\section{Received: 25 March 2019 Accepted: 16 May 2019}

Published online: 04 June 2019

\section{References}

1. Agholme L, Lindström T, Kågedal K, Marcusson J, Hallbeck M (2010) An in vitro model for neuroscience: differentiation of SH-SY5Y cells into cells with morphological and biochemical characteristics of mature neurons. J Alzheimers Dis 20:1069-1082

2. Bading $H$ (2013) Nuclear calcium signalling in the regulation of brain function. Nat Rev Neurosci 14:593-608

3. Blackmore MG, Moore DL, Smith RP, Goldberg JL, Bixby JL, Lemmon VP (2010) High content screening of cortical neurons identifies novel regulators of axon growth. Mol Cell Neurosci 44:43-54

4. Bové J, Martínez-Vicente M, Vila M (2011) Fighting neurodegeneration with rapamycin: mechanistic insights. Nat Rev Neurosci 12:437-452

5. Brewer GJ, Boehler MD, Pearson RA, DeMaris AA, Ide AN, Wheeler BC (2008) Neuron network activity scales exponentially with synapse density. J Neural Eng 6:014001-014008

6. Buchser WJ, Slepak TI, Gutierrez-Arenas O, Bixby JL, Lemmon VP (2010) Kinase/phosphatase overexpression reveals pathways regulating hippocampal neuron morphology. Mol Syst Biol 6:391
7. Calafate S, Buist A, Miskiewicz K, Vijayan V, Daneels G, de Strooper B et al (2015) Synaptic contacts enhance cell-to-cell tau pathology propagation. Cell Rep 11:1176-1183

8. Callif BL, Maunze B, Krueger NL, Simpson MT, Blackmore MG (2017) The application of CRISPR technology to high content screening in primary neurons. Mol Cell Neurosci 80:170-179

9. Cooper DJ, Zunino G, Bixby JL, Lemmon VP (2017) Phenotypic screening with primary neurons to identify drug targets for regeneration and degeneration. Mol Cell Neurosci 80:161-169

10. Cornelissen F, Verstraelen P, Verbeke T, Pintelon I, Timmermans J-P, Nuydens R et al (2013) Quantitation of chronic and acute treatment effects on neuronal network activity using image and signal analysis: toward a high-content assay. J Biomol Screen 18:807-819

11. ]Coyle DE, Li J, Baccei M (2011) Regional differentiation of retinoic acid-induced human pluripotent embryonic carcinoma stem cell neurons. PLoS One 6:e16174

12. Detrez JR, Verstraelen P, Gebuis T, Verschuuren M, Kuijlaars J, Langlois X et al (2016) Image informatics strategies for deciphering neuronal network connectivity. Adv Anat Embryol Cell Biol 219:123-148

13. R Development Core Team R (2008) A language and environment for statistical computing. De Gruyter, Vienna. http://www.R-project.org. Accessed 22 May 2019.

14. Di Z, Klop MJD, Rogkoti V-M, Le Dévédec SE, van de Water B, Verbeek FJ et al (2014) Ultra high content image analysis and phenotype profiling of 3D cultured micro-tissues. PLoS One 9:e109688

15. Dolmetsch R, Geschwind DH (2011) The human brain in a dish: the promise of iPSC-derived neurons. Cell 145:831-834

16. Falke E, Nissanov J, Mitchell TW, Bennett DA, Trojanowski JQ, Arnold SE (2003) Subicular dendritic arborization in Alzheimer's disease correlates with neurofibrillary tangle density. Am J Pathol 163:1615-1621

17. Floyd RA, Carney JM (1992) Free radical damage to protein and DNA: mechanisms involved and relevant observations on brain undergoing oxidative stress. Ann Neurol 32 Suppl:S22-S27

18. Forster Jl, Köglsberger S, Trefois C, Boyd O, Baumuratov AS, Buck L et al (2016) Characterization of differentiated SH-SY5Y as neuronal screening model reveals increased oxidative vulnerability. J Biomol Screen 21:496-509

19. Foster M, Sherrington CS (1897) A textbook of physiology. Macmillan, London

20. Francis JE, Mason D, Levy R (2017) Evaluation of quantum dot conjugated antibodies for immunofluorescent labelling of cellular targets. Beilstein J Nanotechnol 8:1238-1249

21. Frangi AF, Niessen WJ, Vincken KL, Viergever MA (1998) In: Wells WM, Colchester A, Delp S (eds) Multiscale vessel enhancement filtering. Springer Berlin Heidelberg, Berlin, Heidelberg, pp 130-137

22. Ghosh AS, Wang B, Pozniak CD, Chen M, Watts RJ, Lewcock JW (2011) DLK induces developmental neuronal degeneration via selective regulation of proapoptotic JNK activity. J Cell Biol 194:751-764

23. Gordon J, Amini S, White MK (2013) General overview of neuronal cell culture. Methods Mol Biol 1078:1-8

24. Gräff J, Tsai L-H (2013) The potential of HDAC inhibitors as cognitive enhancers. Annu Rev Pharmacol Toxicol 53:311-330

25. Gunhanlar N, Shpak G, van der Kroeg M, Gouty-Colomer LA, Munshi ST, Lendemeijer B et al (2018) A simplified protocol for differentiation of electrophysiologically mature neuronal networks from human induced pluripotent stem cells. Mol Psychiatry 23:1336-1344

26. Gut G, Herrmann MD, Pelkmans L (2018) Multiplexed protein maps link subcellular organization to cellular states. Science 361:eaar7042

27. Haile Y, Fu W, Shi B, Westaway D, Baker G, Jhamandas J et al (2014) Characterization of the NT2-derived neuronal and astrocytic cell lines as alternative in vitro models for primary human neurons and astrocytes. J Neurosci Res 92:1187-1198

28. Harrill JA, Robinette BL, Mundy WR (2011) Use of high content image analysis to detect chemical-induced changes in synaptogenesis in vitro. Toxicol in Vitro 25:368-387

29. Hill E, Jiménez-González C, Tarczyluk M, Nagel DA, Coleman MD, Parri HR (2012) NT2 derived neuronal and astrocytic network signalling. PLoS One 7:e36098

30. A study of gdc-0134 to determine initial safety, tolerability, and pharmacokinetic parameters in participants with amyotrophic lateral sclerosis. 2019. https://www.clinicaltrials.gov/ct2/show/NCT02655614?term= GDC-0134. Accessed 22 May 2019.

31. Hu M, Schurdak ME, Puttfarcken PS, Kouhen El R, Gopalakrishnan M, Li J (2007) High content screen microscopy analysis of a beta 1-42-induced 
neurite outgrowth reduction in rat primary cortical neurons: neuroprotective effects of alpha 7 neuronal nicotinic acetylcholine receptor ligands. Brain Res 1151:227-235

32. Huang Y-WA, Zhou B, Wernig M, SUdhof TC (2017) ApoE2, ApoE3, and ApoE4 differentially stimulate APP transcription and A $\beta$ secretion. Cell 168:427-441.e21

33. Ichikawa M, Muramoto K, Kobayashi K, Kawahara M, Kuroda Y (1993) Formation and maturation of synapses in primary cultures of rat cerebra cortical-cells - an electron-microscopic study. Neurosci Res 16:95-103

34. Jaworski J, Sheng M (2006) The growing role of mTOR in neuronal development and plasticity. Mol Neurobiol 34:205-219

35. Kowalski JW, Gawel M, Pfeffer A, Barcikowska M (2001) The diagnostic value of EEG in Alzheimer disease - correlation with the severity of mental impairment. J Clin Neurophysiol 18:570-575

36. Kuijlaars J, Oyelami T, Diels A, Rohrbacher J, Versweyveld S, Meneghello G et al (2016) Sustained synchronized neuronal network activity in a human astrocyte co-culture system. Sci Rep 6:36529

37. Laplante M, Sabatini DM (2012) mTOR signaling in growth control and disease. Cell 149:274-293

38. Le Pichon CE, Meilandt WJ, Dominguez S, Solanoy H, Lin H, Ngu H et al (2017) Loss of dual leucine zipper kinase signaling is protective in animal models of neurodegenerative disease. Sci Transl Med 9:eaag0394

39. Lin J-R, Fallahi-Sichani M, Sorger PK (2015) Highly multiplexed imaging of single cells using a high-throughput cyclic immunofluorescence method. Nat Commun 6:8390

40. Lin Y-C, Koleske AJ (2010) Mechanisms of synapse and dendrite maintenance and their disruption in psychiatric and neurodegenerative disorders. Annu Rev Neurosci 33:349-378

41. Lipton SA (2006) Paradigm shift in neuroprotection by NMDA receptor blockade: Memantine and beyond. Nat Rev Drug Discov 5:160-170

42. Miller BR, Press C, Daniels RW, Sasaki Y, Milbrandt J, DiAntonio A (2009) A dual leucine kinase-dependent axon self-destruction program promotes Wallerian degeneration. Nat Neurosci 12:387-389

43. Nieland TJF, Logan DJ, Saulnier J, Lam D, Johnson C, Root DE et al (2014) High content image analysis identifies novel regulators of synaptogenesis in a high-throughput RNAi screen of primary neurons. PLoS One 9:e91744

44. O'Rourke NA, Weiler NC, Micheva KD, Smith SJ (2012) Deep molecular diversity of mammalian synapses: why it matters and how to measure it. Nat Rev Neurosci 13:365-379

45. Pani G, De Vos WH, Samari N, de Saint-Georges L, Baatout S, Van Oostveldt $P$ et al (2014) MorphoNeuroNet: an automated method for dense neurite network analysis. Cytometry A 85:188-199

46. Patel S, Cohen F, Dean BJ, La Torre De K, Deshmukh G, Estrada AA et al (2015) Discovery of dual leucine zipper kinase (DLK, MAP3K12) inhibitors with activity in neurodegeneration models. J Med Chem 58:401-418

47. Patel S, Meilandt WJ, Erickson RI, Chen J, Deshmukh G, Estrada AA et al (2017) Selective inhibitors of dual leucine zipper kinase (DLK, MAP3K12) with activity in a model of Alzheimer's disease. J Med Chem 60:8083-8102

48. Patel TP, Man K, Firestein BL, Meaney DF (2015) Automated quantification of neuronal networks and single-cell calcium dynamics using calcium imaging. J Neurosci Methods 243:26-38

49. Pittenger C, Duman RS (2008) Stress, depression, and neuroplasticity: a convergence of mechanisms. Neuropsychopharmacology 33:88-109

50. Pozniak CD, Ghosh AS, Gogineni A, Hanson JE, Lee S-H, Larson JL et al (2013) Dual leucine zipper kinase is required for excitotoxicity-induced neuronal degeneration. J Exp Med 210:2553-2567

51. Reisberg B, Doody R, Stöffler A, Schmitt F, Ferris S, Möbius HJ et al (2003) Memantine in moderate-to-severe Alzheimer's disease. N Engl J Med 348:1333-1341

52. Sabatini DM, Erdjumentbromage H, Lui M, Tempst P, Snyder SH (1994) Raft1 - a mammalian protein that binds to Fkbp12 in a rapamycin-dependent fashion and is homologous to yeast tors. Cell 78:35-43

53. Sharma K, Choi S-Y, Zhang Y, Nieland TJF, Long S, Li M et al (2013) Highthroughput genetic screen for Synaptogenic factors: identification of LRP6 as critical for excitatory synapse Development. Cell Rep 5:1330-1341

54. Siu M, Ghosh AS, Lewcock JW (2018) Dual leucine zipper kinase inhibitors for the treatment of neurodegeneration: Miniperspecteive. J Med Chem 61:8078-8087

55. Spicer TP, Hubbs C, Vaissiere T, Collia D, Rojas C, Kilinc M et al (2018) Improved scalability of neuron-based phenotypic screening assays for therapeutic discovery in neuropsychiatric disorders. Mol Neuropsychiatry 3:141-150

56. Spilman P, Podlutskaya N, Hart MJ, Debnath J, Gorostiza O, Bredesen D et al (2010) Inhibition of mTOR by rapamycin abolishes cognitive deficits and reduces amyloid-beta levels in a mouse model of Alzheimer's disease. PLoS One 5:e9979

57. Stetter O, Battaglia D, Soriano J, Geisel T (2012) Model-free reconstruction of excitatory neuronal connectivity from calcium imaging signals. PLoS Comput Biol 8:e1002653

58. Swiech L, Perycz M, Malik A, Jaworski J (2008) Role of mTOR in physiology and pathology of the nervous system. Biochim Biophys Acta 1784:116-132

59. Taschenberger G, Toloe J, Tereshchenko J, Akerboom J, Wales P, Benz R et al (2013) beta-Synuclein aggregates and induces neurodegeneration in dopaminergic neurons. Ann Neurol 74:109-118

60. Terry RD, Masliah E, Salmon DP, Butters N, DeTeresa R, Hill R et al (1991) Physical basis of cognitive alterations in Alzheimer's disease: synapse loss is the major correlate of cognitive impairment. Ann Neurol 30:572-580

61. Valko M, Leibfritz D, Moncol J, Cronin MTD, Mazur M, Telser J (2007) Free radicals and antioxidants in normal physiological functions and human disease. Int J Biochem Cell Biol 39:44-84

62. Verstraelen P, Detrez JR, Verschuuren M, Kuijlaars J, Nuydens R, Timmermans J-P et al (2017) Dysregulation of microtubule stability impairs Morphofunctional connectivity in primary neuronal networks. Front Cell Neurosci 11:173

63. Verstraelen P, Pintelon I, Nuydens $R$, Cornelissen F, Meert T, Timmermans J-P (2014) Pharmacological characterization of cultivated neuronal networks: relevance to synaptogenesis and synaptic connectivity. Cell Mol Neurobiol 34:757-776

64. Verstraelen $P$, Van Dyck M, Verschuuren M, Kashikar ND, Nuydens R, Timmermans J-P, et al (2018) Image-based profiling of synaptic connectivity in primary neuronal cell culture. Front Neurosci 12

65. Virdee JK, Saro G, Fouillet A, Findlay J, Ferreira F, Eversden S et al (2017) A high-throughput model for investigating neuronal function and synaptic transmission in cultured neuronal networks. Sci Rep 7:14498

66. Watkins TA, Wang B, Huntwork-Rodriguez S, Yang J, Jiang Z, EasthamAnderson J et al (2013) DLK initiates a transcriptional program that couples apoptotic and regenerative responses to axonal injury. Proc Natl Acad Sci U S A 110:4039-4044

67. Welsbie DS, Mitchell KL, Jaskula-Ranga V, Sluch VM, Yang Z, Kim J et al (2017) Enhanced functional genomic screening identifies novel mediators of dual leucine zipper kinase-dependent injury signaling in neurons. Neuron 94:1142-1154.e6

68. Welsbie DS, Yang Z, Ge Y, Mitchell KL, Zhou X, Martin SE et al (2013) Functional genomic screening identifies dual leucine zipper kinase as a key mediator of retinal ganglion cell death. Proc Natl Acad Sci U S A 110:4045-4050

69. Yang S-S, Zhang R, Wang G, Zhang Y-F (2017) The development prospection of HDAC inhibitors as a potential therapeutic direction in Alzheimer's disease. Transl Neurodegener 6:1-6

70. Yarza R, Vela S, Solas M, Ramirez MJ (2016) C-Jun N-terminal kinase (JNK) signaling as a therapeutic target for Alzheimer's disease. Front Pharmacol 6:633-612

71. Yin C, Huang G-F, Sun X-C, Guo Z, Zhang JH (2017) DLK silencing attenuated neuron apoptosis through JIP3/MA2K7/JNK pathway in early brain injury after SAH in rats. Neurobiol Dis 103:133-143

72. Zhang L, Liu C, Wu J, Tao J-J, Sui X-L, Yao Z-G et al (2014) Tubastatin a/ACY1215 improves cognition in Alzheimer's disease transgenic mice. J Alzheimers Dis 41:1193-1205

73. Zhou Q, Lam PY, Han D, Cadenas E (2009) Activation of c-Jun-N-terminal kinase and decline of mitochondrial pyruvate dehydrogenase activity during brain aging. FEBS Lett 583:1132-1140

74. Hsu C-W, Shou D, Huang R, Khuc T, Dai S, Zheng W et al. (2016) Identification of HDAC Inhibitors Using a Cell-Based HDAC I/II Assay. J Biomol Screen. 21:643-652.

75. Parsons CG, Panchenko VA, Pinchenko VO, Tsyndrenko AY, Krishtal OA (1996) Comparative Patch-clamp Studies with Freshly Dissociated Rat Hippocampal and Striatal Neurons on the NMDA Receptor Antagonistic Effects of Amantadine and Memantine. Eur J Neurosci 8:446-454.

76. Ruan B, Pong K, Jow F, Bowlby M, Crozier RA, Liu D et al. (2008) Binding of rapamycin analogs to calcium channels and FKBP52 contributes to their neuroprotective activities. Proc Natl Acad Sci U S A 105:33-38.

77. Zwick V, Simões-Pires CA, Nurisso A, Petit C, Santos Passos Dos C, Randazzo GM et al. (2016) Synthesis of a selective HDAC6 inhibitor active in neuroblasts. Bioorg Med Chem Lett 26:4955-4959. 